\title{
TRACE FORMULAS AND INVERSE SPECTRAL THEORY FOR JACOBI OPERATORS
}

\author{
GERALD TESCHL
}

\begin{abstract}
Based on high energy expansions and Herglotz properties of Green and Weyl $m$-functions we develop a self-contained theory of trace formulas for Jacobi operators. In addition, we consider connections with inverse spectral theory, in particular uniqueness results. As an application we work out a new approach to the inverse spectral problem of a class of reflectionless operators producing explicit formulas for the coefficients in terms of minimal spectral data. Finally, trace formulas are applied to scattering theory with periodic backgrounds.
\end{abstract}

\section{INTRODUCTION}

Trace formulas have a long history in the theory of one-dimensional second order equations. One case of particular importance are periodic potentials. Let

$$
(H f)(n)=a(n) f(n+1)+a(n-1) f(n-1)+b(n) f(n), \quad n \in \mathbb{Z}
$$

be our Jacobi operator with $a(n+N)=a(n), b(n+N)=b(n)$ for some $N \in \mathbb{N}$. Then, using Floquet theory (cf., e.g., [7, Appendix B, [30], 32]) one can show that the spectrum $\sigma(H)$ of $H$ consists of $N$ bands (some of which might collide)

$$
\sigma(H)=\bigcup_{j=1}^{N}\left[E_{2 j-2}, E_{2 j-1}\right], \quad E_{0}<E_{1} \leq E_{2} \cdots<E_{2 N-1} .
$$

Next, we consider finite matrices associated with $H$ obtained by restricting $H$ to finite intervals from $n_{0}$ to $n_{0}+N$ and imposing boundary conditions at the endpoints. Denote the matrix obtained with Dirichlet boundary conditions (i.e., $\left.f\left(n_{0}\right)=0, f\left(n_{0}+N\right)=0\right)$ by $\tilde{H}_{n_{0}}^{\infty}$ and the one obtained with periodic/anti periodic boundary conditions (i.e., $\left.f\left(n_{0}\right)= \pm f\left(n_{0}+N\right), f\left(n_{0}+1\right)= \pm f\left(n_{0}+N+1\right)\right)$ by $\tilde{H}_{n_{0}}^{ \pm}$. The eigenvalues of $\tilde{H}_{n_{0}}^{+}, \tilde{H}_{n_{0}}^{-}$are precisely the even, odd band edges $E_{2 j-2}$, $E_{2 j-1}, 1 \leq j \leq N$, respectively. The eigenvalues of $\tilde{H}_{n}^{\infty}$ are denoted by $\mu_{j}(n)$, $1 \leq j \leq N-1$. Since $\operatorname{tr}\left(\tilde{H}_{n}^{ \pm}\right)=\sum_{j=0}^{N-1} b(n+j)$ and $\operatorname{tr}\left(\tilde{H}_{n}^{\infty}\right)=\sum_{j=1}^{N-1} b(n+j)$ we infer from $b(n)=\operatorname{tr}\left(\tilde{H}_{n}^{ \pm}-\tilde{H}_{n}^{\infty}\right)=\operatorname{tr}\left(\tilde{H}_{n}^{+}+\tilde{H}_{n}^{-}\right) / 2-\operatorname{tr} \tilde{H}_{n}^{\infty}$ by elementary linear algebra

$$
b(n)=\frac{1}{2} \sum_{j=0}^{2 N-1} E_{j}-\sum_{j=1}^{N-1} \mu_{j}(n) .
$$

1991 Mathematics Subject Classification. Primary 39A10, 39A70; Secondary 34B20, 35Q58.

Key words and phrases. Trace formulas, Jacobi operators, inverse spectral theory, scattering theory.

Comm. Math. Phys. 196-1, 175-202 (1998). 
Similarly, considering $\operatorname{tr}\left(\left(\tilde{H}_{n}^{+}\right)^{\ell}+\left(\tilde{H}_{n}^{-}\right)^{\ell}\right) / 2-\operatorname{tr}\left(\tilde{H}_{n}^{\infty}\right)^{\ell}, \ell \in \mathbb{N}$ one can obtain higher order trace relations.

The corresponding formulas for $\ell=1$ (i.e., (1.3) ) and $\ell=2$ were first given in 32. Formula (1.3) plays a key role in the inverse spectral theory of periodic operators and the reconstruction of $a, b$ from suitable spectral data. Those ingredients form the basis for the solution of the periodic initial value problem of the Toda equations (cf., e.g., 77, 10, 39]). Moreover, relation (1.3) was extended to certain reflectionless operators in [2] and successfully used in [2], 22] to solve inverse spectral problems for these operators.

To generalize trace formulas to arbitrary operators one invokes the measure $d \rho_{\delta}$ of $H$ associated with the vector $\delta \in \ell^{2}(\mathbb{Z})$ (cf. Lemma 3.1) by the spectral theorem. Choosing, e.g., $\delta=\delta_{n}$ (the standard basis of $\ell^{2}(\mathbb{Z})$ ) we immediately obtain

$$
\left\langle\delta_{n}, H^{\ell} \delta_{n}\right\rangle=\int_{\mathbb{R}} \lambda^{\ell} d \rho_{\delta_{n}}(\lambda),
$$

connecting the matrix elements $\left\langle\delta_{n}, H^{\ell} \delta_{n}\right\rangle$ with the moments of the measure $d \rho_{\delta_{n}}$. In the special case where $H$ has purely discrete spectrum, the integral can be evaluated,

$$
\left\langle\delta_{n}, H^{\ell} \delta_{n}\right\rangle=\sum_{\lambda \in \sigma(H)} \gamma(\lambda, n, n) \lambda^{\ell},
$$

where $-\gamma(\lambda, n, m)$ is the residue of $G(z, n, m)$ at $z=\lambda \in \sigma(H)$, that is,

$$
\gamma(\lambda, n, n)=\frac{u(\lambda, n) u(\lambda, m)}{\|u(\lambda)\|^{2}}, \quad \lambda \in \sigma(H),
$$

where $u(\lambda)$ is the eigenvector corresponding to $\lambda \in \sigma(H)$. In particular, for $\ell=1$ this gives the interesting result that (for $H$ with purely discrete spectrum) $b(n)$ is equal to the sum over all eigenvalues of $H$ weighted by $\gamma(\lambda, n, n)$.

However, generalizations of $1.3 \mathrm{p}$ cannot be obtained in this way. This can be done by using the exponential measure $\xi d \lambda$ (cf. Appendix A) associated with $d \rho(\lambda)$ as was discovered by F. Gesztesy and B. Simon in [17. There they extended the analog of 1.3 for Schrödinger operators to a much larger class of potentials (in essence, only semiboundedness of the potential is needed) based on the theory of the Krein spectral shift [29. In a subsequent series of papers [18, 19, 21, 25, and [26] they, together with H. Holden and Z. Zhao, exploit the ideas of [17] and extend them in various directions. In [17] they also give a generalization of $(1.3)$ to arbitrary bounded Jacobi operators. However, a comprehensive treatment of trace formulas for Jacobi operators is still missing. Since it is desirable, for further work in inverse spectral theory, to have these powerful tools at one's disposal, one goal of the present paper is to fill this gap.

Furthermore, we want to point out an annoying mismatch in formula 1.3 . In order to express $b(n)$ for all $n \in \mathbb{Z}$ one needs $\left\{E_{j}\right\}_{0 \leq j \leq 2 N-1},\left\{\mu_{j}(n)\right\}_{1 \leq j \leq N}$ for all $n \in \mathbb{Z}$. On the other hand, it is well-known that the spectral data $\left\{E_{j}\right\}_{0 \leq j \leq 2 N-1}$, $\left\{\mu_{j}\left(n_{0}\right)\right\}_{1 \leq j \leq N}$ plus some additional signs $\left\{\sigma_{j}\left(n_{0}\right)\right\}_{1 \leq j \leq N}$ for one fixed $n_{0} \in \mathbb{Z}$ already determine $a(n)^{2}, b(n)$ for all $n \in \mathbb{Z}$. Hence it must be, in principle, possible to express $a(n)^{2}, b(n)$ in terms of these spectral data for one $n_{0} \in \mathbb{Z}$. This naturally raises the question whether one might be able to find explicit expressions of $a(n)^{2}, b(n)$ in terms of suitable minimal spectral data for certain classes of operators. To the best of our knowledge a problem of this kind has not been solved yet. 
Combining the approach of (1.4), the theory of [17, Weyl-Titchmarsh theory, and the moment problem we will add a new wrinkle to the theory of trace formulas and give a solution to this problem for a certain class of bounded reflectionless Jacobi operators in Section 6 .

To give the reader an overview of the results established, we briefly summarize the content of the remaining sections.

Section 2 introduces all the necessary notation and is mainly added to make the paper self-contained and easier to read.

Section 3 contains a comprehensive treatment of asymptotic expansions for Weyl $m$ and Green functions. We establish that expansions for these objects always exist up to arbitrary order. In addition, recursion relations for the expansion coefficients are derived.

Section 4 contains an alternate (recursive) approach to inverse spectral theory which gives simple proofs for standard uniqueness theorems. Moreover, new uniqueness results are established as well.

In Section 5 we derive an infinite series of trace formulas for Jacobi operators in the spirit of 17, 25]. The basic ingredients are the asymptotic expansions of Section 3 and Herglotz properties of these objects. In particular, we extend (1.3) to

((i)) arbitrary order $\ell \in \mathbb{N}$,

((ii)) arbitrary Jacobi operators, and

((iii)) general boundary conditions.

Section 6 applies the results of Section 5 to the theory of reflectionless Jacobi operators, producing formulas of type $(1.3)$ plus an explicit expression of the coefficients $a^{2}, b$ in terms of minimal spectral data.

Section 7 considers scattering theory with periodic backgrounds. Basic objects like transmission and reflection coefficients are introduced. In addition, the analog of a trace formula for Schrödinger operators involving the reflection coefficient is obtained.

Finally, an appendix collects some properties of Herglotz functions needed in the main body of the paper.

\section{Jacobi operators, Resolvents, GreEn's FUnCtions AND ALl that}

Throughout this paper we denote by $\ell(I)=\ell(M, N), I=\{n \in \mathbb{Z} \mid M<n<N\}$, $M, N \in \mathbb{Z} \cup\{ \pm \infty\}$ the set of complex-valued sequences $\{u(n)\}_{n \in I}$ and by $\ell^{p}(I)$, $1 \leq p \leq \infty$ the sequences $u \in \ell(I)$ such that $|u|^{p}$ is summable over $I$. The scalar product in the Hilbert space $\ell^{2}(I)$ will be denoted by

$$
\langle u, v\rangle=\sum_{n \in I} \overline{u(n)} v(n), \quad u, v \in \ell^{2}(I) .
$$

We will be concerned with operators on $\ell^{2}(\mathbb{Z})$ associated with the difference expression

$$
(\tau f)(n)=a(n) f(n+1)+a(n-1) f(n-1)+b(n) f(n),
$$

where $a, b \in \ell(\mathbb{Z})$ satisfy

Hypothesis H.2.1. Suppose

$$
a(n) \in \mathbb{R} \backslash\{0\}, \quad b(n) \in \mathbb{R}, \quad n \in \mathbb{Z} .
$$


If $\tau$ is limit point (l.p.) at both $\pm \infty$ (cf., e.g., [5], 6]), then $\tau$ gives rise to a unique self-adjoint operator $H$ when defined maximally. Otherwise, we need to fix a boundary condition at each endpoint where $\tau$ is limit circle (l.c.) (cf., e.g., [5], [6]). Throughout this paper we denote by $u_{ \pm}(z,),. z \in \mathbb{C}$, nontrivial solutions of $\tau u=z u$ which satisfy the boundary condition at $\pm \infty$ (if any) with $u_{ \pm}(z,.) \in \ell_{ \pm}^{2}(\mathbb{Z})$, respectively. Here $\ell_{ \pm}^{2}(\mathbb{Z})$ denotes the sequences in $\ell(\mathbb{Z})$ being $\ell^{2}$ near $\pm \infty$. The solution $u_{ \pm}(z,$.$) might not exist for z \in \mathbb{R}$ (cf. [37, Lemma A.1), but if it exists it is unique up to a constant multiple.

Picking a fixed $z_{0} \in \mathbb{C} \backslash \mathbb{R}$ we can characterize $H$ by

$$
\begin{aligned}
H: \mathfrak{D}(H) & \rightarrow \ell^{2}(\mathbb{Z}) \\
f & \mapsto \tau f
\end{aligned}
$$

where the domain of $H$ is explicitly given by

$$
\begin{aligned}
& \mathfrak{D}(H)=\left\{f \in \ell^{2}(\mathbb{Z}) \mid \tau f \in \ell^{2}(\mathbb{Z}), \lim _{n \rightarrow+\infty} W_{n}\left(u_{+}\left(z_{0}\right), f\right)=0,\right. \\
& \left.\lim _{n \rightarrow-\infty} W_{n}\left(u_{-}\left(z_{0}\right), f\right)=0\right\}
\end{aligned}
$$

and

$$
W_{n}(f, g)=a(n)(f(n) g(n+1)-f(n+1) g(n))
$$

denotes the (modified) Wronskian. The boundary condition at $\pm \infty$ imposes no additional restriction on $f$ if $\tau$ is l.p. at $\pm \infty$ and can hence be omitted in this case.

Next, consider the sequence

$$
\delta_{n_{0}}^{\beta}=\cos (\alpha) \delta_{n_{0}}+\sin (\alpha) \delta_{n_{0}+1}, \quad \beta=\cot (\alpha), \alpha \in[0, \pi),
$$

where $\delta_{n_{0}}(n)$ is 1 for $n=n_{0}$ and 0 otherwise. Restrict $H$ to the orthogonal complement of $\delta_{n_{0}}^{\beta}$ in $\ell^{2}(\mathbb{Z})$ and denote this restriction by $H_{n_{0}}^{\beta}$, that is,

$$
H_{n_{0}}^{\beta} f=\tau f, \quad f \in \mathfrak{D}\left(H_{n_{0}}^{\beta}\right)=\left\{f \in \mathfrak{D}(H) \mid\left\langle\delta_{n_{0}}^{\beta}, f\right\rangle=0\right\} .
$$

Clearly $H_{n_{0}}^{\beta}$ is self-adjoint on the subspace $\left\{f \in \ell^{2}(\mathbb{Z}) \mid\left\langle\delta_{n_{0}}^{\beta}, f\right\rangle=0\right\}$ but not on $\ell^{2}(\mathbb{Z})$ since $\mathfrak{D}\left(H_{n_{0}}^{\beta}\right)$ is not dense.

Now we turn to resolvents and introduce the Green's function

$$
\begin{aligned}
G(z, m, n) & =\left\langle\delta_{m},(H-z)^{-1} \delta_{n}\right\rangle \\
& =\frac{1}{W\left(u_{-}(z), u_{+}(z)\right)}\left\{\begin{array}{ll}
u_{+}(z, n) u_{-}(z, m) & \text { for } m \leq n \\
u_{+}(z, m) u_{-}(z, n) & \text { for } n \leq m
\end{array},\right.
\end{aligned}
$$

where $z \in \mathbb{C} \backslash \sigma(H)$ and $\sigma(H)$ denotes the spectrum of $H$. For later use we also introduce the convenient abbreviations

$$
\begin{aligned}
g(z, n) & =G(z, n, n)=\frac{u_{+}(z, n) u_{-}(z, n)}{W\left(u_{-}(z), u_{+}(z)\right)} \\
h(z, n) & =2 a(n) G(z, n, n+1)-1 \\
& =\frac{a(n)\left(u_{+}(z, n) u_{-}(z, n+1)+u_{+}(z, n) u_{-}(z, n+1)\right)}{W\left(u_{-}(z), u_{+}(z)\right)} .
\end{aligned}
$$

Similarly, the corresponding object for $H_{n_{0}}^{\beta}$ (viewed as a self-adjoint operator on $\left\{f \in \ell^{2}(\mathbb{Z}) \mid\left\langle\delta_{n_{0}}^{\beta}, f\right\rangle=0\right\}$ ) reads

$$
\begin{aligned}
& G_{n_{0}}^{\beta}(z, m, n)=\left\langle\delta_{m},\left(H_{n_{0}}^{\beta}-z\right)^{-1} \delta_{n}\right\rangle=G(z, m, n)+\gamma^{\beta}\left(z, n_{0}\right)^{-1} \times \\
& \quad\left(G\left(z, m, n_{0}+1\right)+\beta G\left(z, m, n_{0}\right)\right)\left(G\left(z, n_{0}+1, n\right)+\beta G\left(z, n, n_{0}\right)\right),
\end{aligned}
$$


where

$$
\begin{aligned}
\gamma^{\beta}(z, n) & =\frac{\left(u_{+}(z, n+1)+\beta u_{+}(z, n)\right)\left(u_{-}(z, n+1)+\beta u_{-}(z, n)\right)}{W\left(u_{-}(z), u_{+}(z)\right)} \\
& =g(z, n+1)+\frac{\beta}{a(n)} h(z, n)+\beta^{2} g(z, n) .
\end{aligned}
$$

The quantities $g(z, n)$ and $\gamma^{\beta}(z, n)$ are most important for our purpose and satisfy the following recurrence equations which can be verified by tedious but straightforward calculations. We use the shortcuts $\left(f^{-}\right)(n)=f(n-1),\left(f^{+}\right)(n)=f(n+1)$, $\left(f^{++}\right)(n)=f(n+2)$, etc..

Lemma 2.2. Let $u, v$ be two solutions of $\tau u=z u$. Then $g(n)=u(n) v(n)$ satisfies

$$
\frac{\left(a^{+}\right)^{2} g^{++}-a^{2} g}{z-b^{+}}+\frac{a^{2} g^{+}-\left(a^{-}\right)^{2} g^{-}}{z-b}=\left(z-b^{+}\right) g^{+}-(z-b) g,
$$

and

$$
\left(a^{2} g^{+}-\left(a^{-}\right)^{2} g^{-}+(z-b)^{2} g\right)^{2}=(z-b)^{2}\left(W(u, v)^{2}+4 a^{2} g g^{+}\right) .
$$

Moreover, set $\gamma^{\beta}(n)=(u(n+1)+\beta u(n))(v(n+1)+\beta v(n))$, then we have

$$
\begin{aligned}
& \left(\left(a^{+} A^{-}\right)^{2}\left(\gamma^{\beta}\right)^{+}-\left(a^{-} A\right)^{2}\left(\gamma^{\beta}\right)^{-}+B^{2} \gamma^{\beta}\right)^{2} \\
& =\left(A^{-} B\right)^{2}\left(\left(\frac{A}{a} W(u, v)\right)^{2}+4\left(a^{+}\right)^{2} \gamma^{\beta}\left(\gamma^{\beta}\right)^{+}\right),
\end{aligned}
$$

with

$$
\begin{aligned}
A= & a+\beta\left(z-b^{+}\right)+\beta^{2} a^{+} \\
B= & a^{-}\left(z-b^{+}\right)+\beta\left(\left(z-b^{+}\right)(z-b)+a^{+} a^{-}-a^{2}\right) \\
& +\beta^{2} a^{+}(z-b)
\end{aligned}
$$

Remark 2.3. Equations 2.14) and 2.15 are the analogs of well-known differential equations for the diagonal Green function of one-dimensional Schrödinger operators (cf., e.g., [14, 24], equations (5.19) and (5.20)). Equation (2.16) is the analog of equation (5.18) in [24].

Finally, we turn to half line restrictions $H_{ \pm, n_{0}}$ of $H$ defined by

$$
\begin{aligned}
H_{ \pm, n_{0}}: \mathfrak{D}\left(H_{ \pm, n_{0}}\right) & \rightarrow \ell^{2}\left(n_{0}, \pm \infty\right) \\
f & \mapsto \tau f
\end{aligned}
$$

and

$$
\mathfrak{D}\left(H_{ \pm, n_{0}}\right)=\left\{f \in \ell^{2}\left(n_{0}, \pm \infty\right) \mid \tau f \in \ell^{2}\left(n_{0}, \pm \infty\right), \lim _{n \rightarrow \pm \infty} W_{n}\left(u_{ \pm}\left(z_{0}\right), f\right)=0\right\},
$$

where we set $f\left(n_{0}\right)=0$ in the definition of $(\tau f)\left(n_{0} \pm 1\right)$. The corresponding Green functions read

$$
G_{ \pm, n_{0}}(z, m, n)=\frac{ \pm 1}{W\left(s(z), u_{ \pm}(z)\right)} \begin{cases}s\left(z, n, n_{0}\right) u_{ \pm}(z, m) & \text { for } m \geq n \\ s\left(z, m, n_{0}\right) u_{ \pm}(z, n) & \text { for } n \geq m\end{cases}
$$


where $s\left(z, ., n_{0}\right)$ is the solution of $\tau u=z u$ satisfying the Dirichlet boundary condition $s\left(z, n_{0}, n_{0}\right)=0$. The analogous quantities of $g(z, n)$ are the Weyl $m$-functions

$$
\begin{aligned}
m_{ \pm}(z, n) & =\left\langle\delta_{n \pm 1},\left(H_{ \pm, n}-z\right)^{-1} \delta_{n \pm 1}\right\rangle=G_{ \pm, n}(z, n \pm 1, n \pm 1) \\
& =-\frac{u_{ \pm}(z, n \pm 1)}{a\left(n-{ }_{1}^{0}\right) u_{ \pm}(z, n)},
\end{aligned}
$$

which satisfy

$$
a\left(n-{ }_{1}^{0}\right)^{2} m_{ \pm}(z, n)+\frac{1}{m_{ \pm}(z, n \mp 1)}=b(n)-z .
$$

Remark 2.4. We can also consider half line operators $H_{ \pm, n_{0}}^{\beta}$ on $\ell^{2}\left(n_{0}, \pm \infty\right)$ associated with the general boundary condition

$$
f\left(n_{0}+1\right)+\beta f\left(n_{0}\right)=0, \quad \beta \in \mathbb{R} \cup\{\infty\}
$$

at $n_{0}$ rather than only the Dirichlet boundary condition $f\left(n_{0}\right)=0$. We set

$$
H_{+, n_{0}}^{0}=H_{+, n_{0}+1}, \quad H_{+, n_{0}}^{\beta}=H_{+, n_{0}}-a\left(n_{0}\right) \beta^{-1}\left\langle\delta_{n_{0}+1}, .\right\rangle \delta_{n_{0}+1}, \quad \beta \neq 0,
$$

and

$$
H_{-, n_{0}}^{\infty}=H_{-, n_{0}}, \quad H_{-, n_{0}}^{\beta}=H_{-, n_{0}+1}-a\left(n_{0}\right) \beta\left\langle\delta_{n_{0}}, .\right\rangle \delta_{n_{0}}, \quad \beta \neq \infty,
$$

implying $H_{n_{0}}^{\beta} \cong H_{-, n_{0}}^{\beta} \oplus H_{+, n_{0}}^{\beta}$.

\section{Asymptotic EXPAnsions}

In the sequel, asymptotic expansions for $g(z, n)=G(z, n, n)$ and $\gamma^{\beta}(z, n)$ will turn out to be very useful. Both quantities are Herglotz functions as can be seen from

$$
\begin{aligned}
g(z, n) & =\left\langle\delta_{n},(H-z)^{-1} \delta_{n}\right\rangle, \\
\gamma^{\beta}(z, n) & =\left(1+\beta^{2}\right)\left\langle\delta_{n}^{\beta},(H-z)^{-1} \delta_{n}^{\beta}\right\rangle-\frac{\beta}{a(n)}
\end{aligned}
$$

(we note that, by (2.13), $h(z, n)$ is the difference of two Herglotz functions) and the following lemma which is immediate from the spectral theorem.

Lemma 3.1. Suppose $\delta \in \ell^{2}(\mathbb{Z})$ with $\|\delta\|=1$. Then

$$
g(z)=\left\langle\delta,(H-z)^{-1} \delta\right\rangle
$$

is Herglotz, that is,

$$
g(z)=\int_{\mathbb{R}} \frac{1}{\lambda-z} d \rho_{\delta}(\lambda),
$$

where $d \rho_{\delta}(\lambda)=d\left\langle\delta, P_{(-\infty, \lambda]}(H) \delta\right\rangle$ is the spectral measure of $H$ associated to the sequence $\delta$. Moreover,

$$
\operatorname{Im}(g(z))=\operatorname{Im}(z)\left\|(H-z)^{-1} \delta\right\|^{2}
$$

and

$$
g(\bar{z})=\overline{g(z)}, \quad|g(z)| \leq\left\|(H-z)^{-1}\right\| \leq \frac{1}{|\operatorname{Im}(z)|} .
$$

Next, we turn to asymptotic expansions for $g(z, n), h(z, n)$, and $\gamma^{\beta}(z, n)$. 
Theorem 3.2. The quantities $g(z, n), h(z, n)$, and $\gamma^{\beta}(z, n)$ have the following asymptotic expansions for arbitrary $\varepsilon>0$

$$
\begin{aligned}
& g(z, n) \quad \underset{|z| \rightarrow \infty}{\asymp} \quad-\sum_{j=0}^{\infty} \frac{g_{j}(n)}{z^{j+1}}, \quad g_{0}=1, \\
& |\operatorname{Im}(z)| \geq \varepsilon \\
& h(z, n) \underset{|z| \rightarrow \infty}{\asymp} \quad-1-\sum_{j=0}^{\infty} \frac{h_{j}(n)}{z^{j+1}}, \quad h_{0}=0, \\
& |\operatorname{Im}(z)| \geq \varepsilon \\
& \gamma^{\beta}(z, n) \quad \underset{|z| \rightarrow \infty}{\asymp} \quad-\frac{\beta}{a(n)}-\sum_{j=0}^{\infty} \frac{\gamma_{j}^{\beta}(n)}{z^{j+1}}, \quad \gamma_{0}^{\beta}=1+\beta^{2} . \\
& |\operatorname{Im}(z)| \geq \varepsilon
\end{aligned}
$$

Moreover, the coefficients are given by

$$
\begin{aligned}
g_{j}(n) & =\left\langle\delta_{n}, H^{j} \delta_{n}\right\rangle, \quad j \in \mathbb{N}_{0}, \\
h_{j}(n) & =2 a(n)\left\langle\delta_{n+1}, H^{j} \delta_{n}\right\rangle, \quad j \in \mathbb{N}_{0}, \\
\gamma_{j}^{\beta}(n) & =\left\langle\left(\delta_{n+1}+\beta \delta_{n}\right), H^{j}\left(\delta_{n+1}+\beta \delta_{n}\right)\right\rangle \\
& =g_{j}(n+1)+\frac{\beta}{a(n)} h_{j}(n)+\beta^{2} g_{j}(n), \quad j \in \mathbb{N}_{0} .
\end{aligned}
$$

Proof. We only carry out the proof for $g(z, n)$ since the remaining expansions are similar. Rewriting $g(z, n)$ as

$$
\begin{aligned}
g(z, n) & =\left\langle\delta_{n},(H-z)^{-1} \delta_{n}\right\rangle \\
& =-\sum_{j=0}^{N-1} \frac{\left\langle\delta_{n}, H^{j} \delta_{n}\right\rangle}{z^{j+1}}+\frac{1}{z^{N}}\left\langle\delta_{n}, H^{N}(H-z)^{-1} \delta_{n}\right\rangle, N \in \mathbb{N}
\end{aligned}
$$

shows that it suffices to vindicate that the last term is $O\left(z^{-N}\right)$. This follows from

$$
\left|\left\langle\delta_{n}, H^{N}(H-z)^{-1} \delta_{n}\right\rangle\right| \leq \frac{\left\|H^{N} \delta_{n}\right\|}{|\operatorname{Im}(z)|} \leq \frac{\left\|H^{N} \delta_{n}\right\|}{\varepsilon} .
$$

Remark 3.3. (i). If $H$ is bounded, the above expansions are in fact Laurent series converging for $|z|>\|H\|$.

(ii). Pick $\varepsilon(n) \in\{-1,+1\}$ and introduce $a_{\varepsilon}(n)=\varepsilon(n) a(n)$ and $b_{\varepsilon}(n)=b(n)$. Then the operator $H_{\varepsilon}$ associated with $a_{\varepsilon}, b_{\varepsilon}$ is unitarily equivalent to $H$. Indeed, take the unitary operator $U_{\varepsilon}=\left\{\tilde{\varepsilon}(n) \delta_{m, n}\right\}_{m, n \in \mathbb{Z}}$, where $\varepsilon(n+1) \tilde{\varepsilon}(n)=\varepsilon(n)$, then $H_{\varepsilon}=U_{\varepsilon} H U_{\varepsilon}^{-1}$. In particular, this shows that $g(n), h(n)$ do not depend on the sign of $a$, that is, they only depend on $a^{2}$.

The following lemma ([7], Lemma 2.1) shows how to compute $g_{j}, h_{j}$ recursively.

Lemma 3.4. The coefficients $g_{j}(n)$ and $h_{j}(n)$ for $j \in \mathbb{N}_{0}$ satisfy the following recursion relation

$$
\begin{aligned}
g_{j+1} & =\frac{h_{j}+h_{j}^{-}}{2}+b g_{j}, \\
h_{j+1}-h_{j+1}^{-} & =2\left(a^{2} g_{j}^{+}-\left(a^{-}\right)^{2} g_{j}^{-}\right)+b\left(h_{j}-h_{j}^{-}\right) .
\end{aligned}
$$


Proof. The first equation follows from

$$
g_{j+1}(n)=\left\langle H \delta_{n}, H^{j} \delta_{n}\right\rangle=\frac{h_{j}(n)+h_{j}(n-1)}{2}+b(n) g_{j}(n) .
$$

Similarly,

$$
h_{j+1}(n)=b(n) h_{j}(n)+2 a(n)^{2} g_{j}(n+1)+2 a(n-1) a(n)\left\langle\delta_{n+1}, H^{j} \delta_{n-1}\right\rangle
$$

Eliminating $\left\langle\delta_{n+1}, H^{j} \delta_{n-1}\right\rangle$ completes the proof.

This system does not determine $g_{j}(n), h_{j}(n)$ uniquely since it requires solving a first-order recurrence relation at each step, producing an unknown summation constant each time. To determine these constants we assign the weight one to $a(n)$ and $b(n), n \in \mathbb{Z}$. Then $g_{j+1}(n)$ and $h_{j}(n)$ have weight $j+1$, fixing the summation constants.

To avoid this drawback we advocate a different approach using (2.15). First observe that $h_{j}(n)$ can be determined if $g_{j}(n)$ is known using

$$
h_{j+1}=b h_{j}+g_{j+2}-2 b g_{j+1}+a^{2} g_{j}^{+}-\left(a^{-}\right)^{2} g_{j}^{-}+b^{2} g_{j}, \quad j \in \mathbb{N}_{0},
$$

which follows after inserting (3.15) into (3.16). In addition, inserting the expansion (3.7) for $g(z, n)$ into 2.15 and comparing coefficients of $z^{j}$ one infers

$$
\begin{aligned}
& g_{0}=1, \quad g_{1}=b, \quad g_{2}=a^{2}+\left(a^{-}\right)^{2}+b^{2}, \\
& g_{3}=a^{2}\left(b^{+}+2 b\right)+\left(a^{-}\right)^{2}\left(2 b+b^{-}\right)+b^{3},
\end{aligned}
$$

and

$$
\begin{aligned}
g_{j+1}= & 2 b g_{j}-a^{2} g_{j-1}^{+}+\left(a^{-}\right)^{2} g_{j-1}^{-}-b^{2} g_{j-1}-\frac{1}{2} \sum_{\ell=0}^{j-1} k_{j-\ell-1} k_{\ell} \\
& +2 a^{2}\left(\sum_{\ell=0}^{j-1} g_{j-\ell-1} g_{\ell}^{+}-2 b \sum_{\ell=0}^{j-2} g_{j-\ell-2} g_{\ell}^{+}+b^{2} \sum_{\ell=0}^{j-3} g_{j-\ell-3} g_{\ell}^{+}\right),
\end{aligned}
$$

for $j \geq 3$, where $k_{0}(n)=-b(n)$ and

$$
k_{j}=a^{2} g_{j-1}^{+}-\left(a^{-}\right)^{2} g_{j-1}^{-}+b^{2} g_{j-1}-2 b g_{j}+g_{j+1}, \quad j \in \mathbb{N} .
$$

Analogously, one can get a recurrence relation for $\gamma_{j}^{\beta}$ using (2.16). Since this approach gets too cumbersome we omit further details at this point but note that $\gamma_{j}^{\beta}$ can be computed from 3.12 . Invoking 3.19 one explicitly obtains

$$
h_{0}=0, \quad h_{1}=2 a^{2}, \quad h_{2}=2 a^{2}\left(b^{+}+b\right)
$$

and hence

$$
\begin{aligned}
& \gamma_{0}^{\beta}=1+\beta^{2}, \quad \gamma_{1}^{\beta}=b^{+}+2 a \beta+b \beta^{2}, \\
& \gamma_{2}^{\beta}=\left(a^{+}\right)^{2}+a^{2}+\left(b^{+}\right)^{2}+2 a\left(b^{+}+b\right) \beta+\left(a^{2}+\left(a^{-}\right)^{2}+b^{2}\right) \beta^{2} .
\end{aligned}
$$

Remark 3.5. Instead of (3.19) and 3.21 one can also use 3.15) and

$$
h_{j+1}=2 a^{2} \sum_{\ell=0}^{j} g_{j-\ell} g_{\ell}^{+}-\frac{1}{2} \sum_{\ell=0}^{j} h_{j-\ell} h_{\ell}, \quad j \in \mathbb{N},
$$

together with (3.15) to determine $g_{j}, h_{j}$. The above equation follows as before using (4.6) below. 
Next we turn to Weyl $m$-functions. As before we obtain

Lemma 3.6. The quantities $m_{ \pm}(z, n)$ have the asymptotic expansions

$$
m_{ \pm}(z, n) \underset{\substack{|z| \rightarrow \infty \\|\operatorname{Im}(z)| \geq \varepsilon}}{\asymp}-\sum_{j=0}^{\infty} \frac{m_{ \pm, j}(n)}{z^{j+1}}, \quad m_{ \pm, 0}(n)=1 .
$$

The coefficients $m_{ \pm, j}(n)$ are given by

$$
m_{ \pm, j}(n)=\left\langle\delta_{n \pm 1},\left(H_{ \pm, n}\right)^{j} \delta_{n \pm 1}\right\rangle, \quad j \in \mathbb{N}
$$

and satisfy

$$
\begin{aligned}
& m_{ \pm, 0}=1, \quad m_{ \pm, 1}=b^{ \pm}, \\
& m_{ \pm, j+1}=b^{ \pm} m_{ \pm, j}+\frac{\left(a^{+}\right)^{2}}{\left(a^{--}\right)^{2}} \sum_{\ell=0}^{j-1} m_{ \pm, j-\ell-1} m_{ \pm, \ell}^{+}, \quad j \in \mathbb{N} .
\end{aligned}
$$

Remark 3.7. As in Remark 3.3 we have that (3.26) converges for $|z|>\left\|H_{ \pm, n}\right\|$ if $H_{ \pm, n}$ is bounded and $m_{ \pm}(z, n)$ depend only on $a^{2}$.

\section{INVERSE SPECTRAL THEORY}

In this section we present a simple recursive method of reconstructing the sequences $a^{2}, b$ when the Weyl matrix

$$
\begin{aligned}
M(z, n) & =\left(\begin{array}{cc}
G(z, n, n) & G(z, n+1, n) \\
G(z, n, n+1) & G(z, n+1, n+1)
\end{array}\right)-\frac{1}{2 a(n)}\left(\begin{array}{ll}
0 & 1 \\
1 & 0
\end{array}\right) \\
& =\left(\begin{array}{cc}
g(z, n) & \frac{h(z, n)}{2 a(n)} \\
\frac{h(z, n)}{2 a(n)} & g(z, n+1)
\end{array}\right), \quad z \in \mathbb{C} \backslash \sigma(H)
\end{aligned}
$$

is known for one fixed $n \in \mathbb{Z}$. As a consequence, we are led to several uniqueness results.

From the previous section we know

$$
\begin{aligned}
& g(z, n)=-\frac{1}{z}-\frac{b(n)}{z^{2}}+O\left(\frac{1}{z^{3}}\right), \\
& h(z, n)=-1-\frac{2 a(n)^{2}}{z^{2}}+O\left(\frac{1}{z^{3}}\right) .
\end{aligned}
$$

Here and in the remainder of this paper all $O\left(\frac{1}{z^{\ell}}\right)$ terms apply for $|z| \rightarrow \infty,|\operatorname{Im}(z)| \geq$ $\varepsilon>0$. Hence

$$
\begin{aligned}
b(n) & =-\lim _{z \rightarrow \mathrm{i} \infty} z(1+z g(z, n)), \\
a(n)^{2} & =-\frac{1}{2} \lim _{z \rightarrow \mathrm{i} \infty} z^{2}(1+h(z, n)) .
\end{aligned}
$$

Moreover, we have the useful identities (use 2.10) and (2.11)

$$
4 a(n)^{2} g(z, n) g(z, n+1)=h(z, n)^{2}-1
$$

and

$$
h(z, n+1)+h(z, n)=2(z-b(n+1)) g(z, n+1),
$$

which show that $g(z, n)$ and $h(z, n)$ together with $a(n)^{2}$ and $b(n)$ can be determined recursively if, say, $g\left(z, n_{0}\right)$ and $h\left(z, n_{0}\right)$ are given. 
In addition, we infer that $a(n)^{2}, g(z, n), g(z, n+1)$ determine $h(z, n)$ up to one sign,

$$
h(z, n)=\left(1+4 a(n)^{2} g(z, n) g(z, n+1)\right)^{1 / 2}
$$

since $h(z, n)$ is holomorphic with respect to $z \in \mathbb{C} \backslash \sigma(H)$ and $\overline{h(z, n)}=h(\bar{z}, n)$. However, this sign can be determined from the asymptotic behavior $h(z, n)=$ $-1+O\left(z^{-2}\right)$.

Hence we have reproved the well-known result that $M\left(z, n_{0}\right)$ determines the sequences $a^{2}, b$. In fact, we have proved the slightly stronger result:

Theorem 4.1. One of the following set of data

(i) $g\left(., n_{0}\right)$ and $h\left(., n_{0}\right)$

(ii) $g\left(., n_{0}+1\right)$ and $h\left(., n_{0}\right)$

(iii) $g\left(., n_{0}\right), g\left(., n_{0}+1\right)$, and $a\left(n_{0}\right)^{2}$

for one fixed $n_{0} \in \mathbb{Z}$ uniquely determines the sequences $a^{2}$ and $b$.

Remark 4.2. (i) We want to emphasize that the diagonal elements $g\left(z, n_{0}\right)$ and $g\left(z, n_{0}+1\right)$ alone plus $a\left(n_{0}\right)^{2}$ are sufficient to reconstruct $a(n)^{2}, b(n)$. This is in contradistinction to the case of one-dimensional Schrödinger operators, where the diagonal elements of the Weyl matrix determine the potential only up to reflection. It is not clear to me whether this different behavior of Jacobi operators has been previously noted in the literature.

The reader might wonder how the Weyl matrix of the operator $H_{R}$ associated with the $\left(a t n_{0}\right)$ reflected coefficients $a_{R}, b_{R}$ (i.e., $a_{R}\left(n_{0}-k-1\right)=a\left(n_{0}+k\right)$, $\left.b_{R}\left(n_{0}-k\right)=b\left(n_{0}+k\right), k \in \mathbb{Z}\right)$ look like. Since reflection at $n_{0}$ exchanges $m_{ \pm}\left(z, n_{0}\right)$ (i.e., $m_{R, \pm}\left(z, n_{0}\right)=m_{\mp}\left(z, n_{0}\right)$ ) we infer

$$
\begin{aligned}
g_{R}\left(z, n_{0}\right)= & g\left(z, n_{0}\right), \\
h_{R}\left(z, n_{0}\right)= & -h\left(z, n_{0}\right)+2\left(z-b\left(n_{0}\right)\right) g\left(z, n_{0}\right), \\
g_{R}\left(z, n_{0}+1\right)= & \frac{a\left(n_{0}\right)^{2}}{a\left(n_{0}-1\right)^{2}} g\left(z, n_{0}+1\right)+\frac{z-b\left(n_{0}\right)}{a\left(n_{0}-1\right)^{2}}\left(h\left(z, n_{0}\right)\right. \\
& \left.+\left(z-b\left(n_{0}\right)\right) g\left(z, n_{0}\right)\right)
\end{aligned}
$$

in obvious notation.

(ii) Remark 3.3(ii) shows that the sign of a(n) cannot be determined from either $g\left(z, n_{0}\right), h\left(z, n_{0}\right)$, or $g\left(z, n_{0}+1\right)$.

(iii). Clearly, if $H$ is l.c. at $\pm \infty$ the corresponding boundary condition is determined by $M(z, n)$ as well.

(iv). Equation (4.6) is equivalent to $\operatorname{det} M(z, n)=-1 /(2 a(n))^{2}$. The analogous equation for the Schrödinger case was first used by Rofe-Beketov in connection with inverse problems (see 31, Section 7.3).

The off diagonal Green function can be recovered as follows

$$
G(z, n, n+k)=g(z, n) \prod_{j=n}^{n+k-1} \frac{1+h(z, j)}{2 a(j) g(z, j)}, \quad k>0,
$$

and we remark

$$
\begin{aligned}
& a(n)^{2} g(z, n+1)-a(n-1)^{2} g(z, n-1)+(z-b(n))^{2} g(z, n) \\
& \quad=(z-b(n)) h(z, n) .
\end{aligned}
$$


A similar procedure works for $H_{+}$. The asymptotic expansion

$$
m_{+}(z, n)=-\frac{1}{z}-\frac{b(n+1)}{z^{2}}-\frac{a(n+1)^{2}+b(n+1)^{2}}{z^{3}}+O\left(z^{-4}\right)
$$

shows that $a(n+1)^{2}, b(n+1)$ can be recovered from $m_{+}(z, n)$. In addition, 2.23) shows that $m_{+}\left(z, n_{0}\right)$ determines $a(n)^{2}, b(n), m_{+}(z, n), n>n_{0}$. Similarly, (by reflection) $m_{-}\left(z, n_{0}\right)$ determines $a(n-1)^{2}, b(n), m_{-}(z, n-1), n<n_{0}$. Hence both $m_{ \pm}\left(z, n_{0}\right)$ determine $a(n)^{2}, b(n)$ except for $a\left(n_{0}-1\right)^{2}, a\left(n_{0}\right)^{2}, b\left(n_{0}\right)$. However, introducing $\tilde{m}_{ \pm}(z, n)=\mp u_{ \pm}(z, n+1) /\left(a(n) u_{ \pm}(z, n)\right)$ and considering

$$
\tilde{m}_{+}(z, n)=m_{+}(z, n), \quad \tilde{m}_{-}(z, n)=\frac{z-b(n)+a(n-1)^{-2} m_{-}(z, n)}{a(n)^{2}}
$$

we see that $\tilde{m}_{-}\left(z, n_{0}\right)$ determines $a\left(n_{0}-1\right)^{2}, a\left(n_{0}\right)^{2}, b\left(n_{0}\right)$ and $m_{-}\left(z, n_{0}\right)$. Summarizing:

Theorem 4.3. The quantities $\tilde{m}_{ \pm}\left(z, n_{0}\right)$ uniquely determine $a(n)^{2}, b(n)$ for all $n \in \mathbb{Z}$. Moreover, we have

$$
\begin{aligned}
g(z, n)=\frac{-a(n)^{-2}}{\tilde{m}_{+}(z, n)+\tilde{m}_{-}(z, n)}, \quad g(z, n+1) & =\frac{\tilde{m}_{+}(z, n) \tilde{m}_{-}(z, n)}{\tilde{m}_{+}(z, n)+\tilde{m}_{-}(z, n)}, \\
h(z, n) & =\frac{\tilde{m}_{+}(z, n)-\tilde{m}_{-}(z, n)}{\tilde{m}_{+}(z, n)+\tilde{m}_{-}(z, n)},
\end{aligned}
$$

and conversely

$$
\tilde{m}_{ \pm}(z, n)=\frac{1 \pm h(z, n)}{2 a(n)^{2} g(z, n)}=-\frac{2 g(z, n+1)}{1 \mp h(z, n)} .
$$

Next we recall the function $\gamma^{\beta}(z, n)$ introduced in 2.13 with asymptotic expansion

$$
\gamma^{\beta}(z, n)=-\frac{\beta}{a(n)}-\frac{1+\beta^{2}}{z}-\frac{b(n+1)+2 \beta a(n)+\beta^{2} b(n)}{z^{2}}+O\left(\frac{1}{z^{3}}\right) .
$$

Our goal is to prove

Theorem 4.4. Let $\beta_{1,2} \in \mathbb{R} \cup\{\infty\}$ with $\beta_{1} \neq \beta_{2}$ be given. Then $\gamma^{\beta_{j}}\left(., n_{0}\right)$, $j=1,2$ for one fixed $n_{0} \in \mathbb{Z}$ uniquely determines $a(n)^{2}, b(n)$ for all $n \in \mathbb{Z}$ (set $\left.\gamma^{\infty}(z, n)=g(z, n)\right)$ unless $\left(\beta_{1}, \beta_{2}\right)=(0, \infty),(\infty, 0)$. In the latter case $a\left(n_{0}\right)^{2}$ is needed in addition. More explicitly, we have

$$
\begin{aligned}
g(z, n) & =\frac{\gamma^{\beta_{1}}(z, n)+\gamma^{\beta_{2}}(z, n)+2 R(z)}{\left(\beta_{2}-\beta_{1}\right)^{2}} \\
g(z, n+1) & =\frac{\beta_{2}^{2} \gamma^{\beta_{1}}(z, n)+\beta_{1}^{2} \gamma^{\beta_{2}}(z, n)+2 \beta_{1} \beta_{2} R(z)}{\left(\beta_{2}-\beta_{1}\right)^{2}}, \\
h(z, n) & =\frac{\beta_{2} \gamma^{\beta_{1}}(z, n)+\beta_{1} \gamma^{\beta_{2}}(z, n)+\left(\beta_{1}+\beta_{2}\right) R(z)}{(-2 a(n))^{-1}\left(\beta_{2}-\beta_{1}\right)^{2}},
\end{aligned}
$$

where $R(z)$ is the branch of

$$
R(z)=\left(\frac{\left(\beta_{2}-\beta_{1}\right)^{2}}{4 a(n)^{2}}+\gamma^{\beta_{1}}(z, n) \gamma^{\beta_{2}}(z, n)\right)^{1 / 2}=\frac{\beta_{1}+\beta_{2}}{2 a(n)}+O\left(\frac{1}{z}\right),
$$


which is holomorphic for $z \in \mathbb{C} \backslash \mathbb{R}$ and has asymptotic behavior as indicated. If one of the numbers $\beta_{1,2}$ equals $\infty$, one has to replace all formulas by their limit using $g(z, n)=\lim _{\beta \rightarrow \infty} \beta^{-2} \gamma^{\beta}(z, n)$.

Proof. Clearly, if $\left(\beta_{1}, \beta_{2}\right) \neq(0, \infty),(\infty, 0)$ we can determine $a(n)$ from 4.18. Hence by Theorem 4.1 it suffices to show 4.19 - 4.21). Since 4.19 follows from (4.6) and the other two, it remains to establish 4.20) and 4.21). This will follow if we prove that the system

$$
\left(g^{+}\right)^{2}+2 \frac{\beta_{j}}{2 a(n)} h g^{+}+\frac{\beta_{j}^{2}}{4 a(n)^{2}}\left(h^{2}-1\right)=g^{+} \gamma^{\beta_{j}}(z, n), \quad j=1,2
$$

has a unique solution $\left(g^{+}, h\right)=(g(z, n+1), h(z, n))$ for $|z|$ large enough, $|\operatorname{Im}(z)| \geq \varepsilon$, which is holomorphic with respect to $z$ and satisfies the asymptotic requirements from above. We first consider the case $\beta_{j} \neq 0, \infty$. Changing to new variables $\left(x_{1}, x_{2}\right), x_{j}=\left(2 a(n) / \beta_{j}\right) g^{+}+h$, our system reads

$$
x_{j}^{2}-1=\frac{\beta_{1} \beta_{2}}{\beta_{j}^{2}} \frac{2 a(n) \gamma^{\beta_{j}}(z, n)}{\beta_{2}-\beta_{1}}\left(x_{1}-x_{2}\right), \quad j=1,2 .
$$

Picking $|z|$ large enough we can assume $\gamma^{\beta_{j}}(z, n) \neq 0$ and the solution set of the new system is given by the intersection of two parabolas. In particular, 4.23 has at most four solutions. Two of them are clearly $g^{+}=0, \quad h= \pm 1$. But they do not have the correct asymptotic behavior and hence are of no interest to us. The remaining two solutions are given by 4.20) and (4.21) with the branch of $R(z)$ arbitrarily. However, we only get correct asymptotics $\left(g^{+}=-z^{-1}+O\left(z^{-2}\right)\right.$ resp. $\left.h=-1+O\left(z^{-2}\right)\right)$ if we fix the branch as in 4.22 . This shows that $g(z, n+1), h(z, n)$ can be reconstructed from $\gamma^{\beta_{j}}, j=1,2$ and we are done. The remaining cases can be treated similarly.

Corollary 4.5. Suppose $H$ has purely discrete spectrum. Then a $\left(n_{0}\right), \sigma(H)$ plus $\beta_{j}, \sigma\left(H_{n_{0}}^{\beta_{j}}\right), j=1,2$ for two values $\beta_{1} \neq \beta_{2}$ uniquely determine the coefficients $a(n)^{2}, b(n)$ (and the boundary condition at $\pm \infty$ if any).

Proof. Since $H$ has purely discrete spectrum the same is true for $H_{n_{0}}^{\beta}$. Hence $\gamma^{\beta}\left(z, n_{0}\right)$ is meromorphic with poles at the eigenvalues of $H$ and zeros at the eigenvalues of $H_{n_{0}}^{\beta}$ following from 2.13 (if eigenvalues of $H$ and $H_{n_{0}}^{\beta}$ coincide we have a double zero in numerator of (2.13) and a single zero in the denominator). Thus we know when $\gamma^{\beta}\left(z, n_{0}\right)$ changes sign implying that we know the exponential Herglotz measure of $\gamma^{\beta}\left(z, n_{0}\right)$ (cf. A.2). The remaining constant $c$ in A.2 follows from the asymptotic behavior (see also 5.19$)$ ). Hence we can reconstruct $\gamma^{\beta}\left(z, n_{0}\right)$ from $a\left(n_{0}\right), \sigma(H)$ and $\beta, \sigma\left(H_{n_{0}}^{\beta}\right)$ completing the proof.

Finally, let us turn to half line operators $H_{+}^{\beta}=H_{+, 0}^{\beta}$ (cf. Remark 2.4). Since the dependence one $a(0)$ can be removed by scaling $\beta$, we assume without restriction $a(0)=1$ for the remainder of this section. We will now prove the following generalization of a result by Fu and Hochstadt [13] (where the special case $\beta_{1}=0, \beta_{2}=\infty$ is proved under somewhat more restrictive conditions).

Theorem 4.6. Suppose the spectrum of $H_{+}^{\beta}$ is purely discrete for one $\beta \in \mathbb{R} \cup\{\infty\}$ (and hence for all $\beta$ ) and let $\beta_{j}, j=1,2$ be two different values which have opposite 
signs if $0<\left|\beta_{j}\right|<\infty$. Then $\beta_{j}$ plus $\sigma\left(H_{+}^{\beta_{j}}\right), j=1,2$ uniquely determine the coefficients $a(n)^{2}, b(n)$ (and the boundary condition at $+\infty$ if any).

Proof. Without restriction we suppose $\beta_{2} \neq 0$ and $\beta_{1} \neq \infty$. Then

$$
F(z)=\frac{\beta_{2}\left(m_{+}(z)+\beta_{1}\right)}{m_{+}(z)+\beta_{2}}=\frac{-1}{\frac{-1}{\beta_{2}}-\frac{1}{m_{+}(z)}}+\frac{\beta_{1} \beta_{2}}{m_{+}(z)+\beta_{2}}
$$

is a meromorphic Herglotz function since $m_{+}(z)=m_{+}(z, 0)$ is. Moreover, since $m_{+}(z)=\frac{u_{+}(z, 1)}{u_{+}(z, 0)}\left(\right.$ where $u_{+}(z, 0)$ has to be defined as $-a(1) u_{+}(z, 2)+(z-b(1)) u(z, 1)$ recall our convention $a(0)=1$ ), we infer that the zeros of $F(z)$ are given by the eigenvalues of $H_{+}^{\beta_{1}}$ and the poles by the eigenvalues of $H_{+}^{\beta_{2}}$. Thus we know the exponential Herglotz measure $\xi(\lambda)$ of $F(z)$ (cf. A.2). The remaining constant $c$ in A.2 can be determined from the asymptotic behavior $F(z)=\beta_{1}-(1-$ $\left.\beta_{1} \beta_{2}^{-1}\right) z^{-1}+O\left(z^{-2}\right)$. Thus $F(z)$ is known and solving $F(z)$ for $m_{+}(z)$ finishes the proof.

\section{General trace formulas and $\xi$ Functions}

In this section we will investigate trace formulas for Jacobi operators $H$. We will essentially follow the philosophy of [17, 25] and use the exponential Herglotz representation A.2 rather than A.1). This will produce generalizations of the formula (1.3).

To avoid the Abelian limits of [17] we will first consider the case where $H$ (and thus $a, b)$ is bounded. We abbreviate

$$
E_{0}=\inf \sigma(H), \quad E_{\infty}=\sup \sigma(H),
$$

and note that $G(\lambda, n, n)>0$ for $\lambda<E_{0}$, which follows from $(H-\lambda)>0$ (implying $\left.(H-\lambda)^{-1}>0\right)$. Similarly, $G(\lambda, n, n)<0$ for $\lambda>E_{\infty}$, following from $(H-\lambda)<0$. Our main tool will be the following exponential representation of the Herglotz function $g(z, n)=G(z, n, n)$ (cf. Theorem A.2

$$
g(z, n)=|g(\mathrm{i}, n)| \exp \left(\int_{\mathbb{R}}\left(\frac{1}{\lambda-z}-\frac{\lambda}{1+\lambda^{2}}\right) \xi(\lambda, n) d \lambda\right), \quad z \in \mathbb{C} \backslash \sigma(H),
$$

where the $\xi$ function $\xi(\lambda, n)$ is defined by

$$
\xi(\lambda, n)=\frac{1}{\pi} \lim _{\varepsilon \downarrow 0} \arg g(\lambda+\mathrm{i} \varepsilon, n), \quad \arg (.) \in(-\pi, \pi] .
$$

In addition, $\xi(\lambda, n)$ (which is only defined a.e.) satisfies $0 \leq \xi(\lambda, n) \leq 1$,

$$
\int_{\mathbb{R}} \frac{\xi(\lambda, n)}{1+\lambda^{2}} d \lambda=\arg g(\mathrm{i}, n), \text { and } \xi(\lambda, n)=\left\{\begin{array}{l}
0 \text { for } z<E_{0} \\
1 \text { for } z>E_{\infty}
\end{array} .\right.
$$

Using (5.4) together with the asymptotic behavior of $g(., n)$ we infer

$$
g(z, n)=\frac{1}{E_{\infty}-z} \exp \left(\int_{E_{0}}^{E_{\infty}} \frac{\xi(\lambda, n) d \lambda}{\lambda-z}\right) .
$$

Theorem 5.1. Suppose $H$ is bounded and let $\xi(\lambda, n)$ be defined as above. Then we have the following trace formula

$$
b^{(\ell)}(n)=E_{\infty}^{\ell}-\ell \int_{E_{0}}^{E_{\infty}} \lambda^{\ell-1} \xi(\lambda, n) d \lambda,
$$


where

$$
\begin{aligned}
& b^{(1)}(n)=b(n), \\
& b^{(\ell)}(n)=\ell g_{\ell}(n)-\sum_{j=1}^{\ell-1} g_{\ell-j}(n) b^{(j)}(n), \quad \ell>1 .
\end{aligned}
$$

Proof. The claim follows after expanding both sides of

$$
\ln \left(\left(E_{\infty}-z\right) g(z, n)\right)=\int_{E_{0}}^{E_{\infty}} \frac{\xi(\lambda, n) d \lambda}{\lambda-z}
$$

and comparing coefficients using the following connections between the series of $g(z)$ and $\ln (1+g(z))$ (cf., e.g., [33]). Let $g(z)$ have the asymptotic expansion

$$
g(z)=\sum_{\ell=1}^{\infty} \frac{g_{\ell}}{z^{\ell}}
$$

as $z \rightarrow \infty$. Then we have

$$
\ln (1+g(z))=\sum_{\ell=1}^{\infty} \frac{c_{\ell}}{z^{\ell}}
$$

where

$$
c_{1}=g_{1}, \quad c_{\ell}=g_{\ell}-\sum_{j=1}^{\ell-1} \frac{j}{\ell} g_{\ell-j} c_{j}, \quad \ell \geq 2 .
$$

We remark that the special case $\ell=1$ of equation 5.6

$$
b(n)=E_{\infty}-\int_{E_{0}}^{E_{\infty}} \xi(\lambda, n) d \lambda=\frac{E_{0}+E_{\infty}}{2}+\frac{1}{2} \int_{E_{0}}^{E_{\infty}}(1-2 \xi(\lambda, n)) d \lambda
$$

has first been given in 17 .

Next we turn to unbounded operators. In order to avoid Abelian limits here as well, we resort to a little trick. This will also show how our investigations tie in with the theory of Krein [29] and rank one perturbations (see also [17, Appendix A, 19, 34]). Consider

$$
H_{n, \theta}=H+\theta\left\langle\delta_{n}, .\right\rangle \delta_{n}, \quad \theta \geq 0 .
$$

Then, as in [17, Appendix A, one computes

$$
\operatorname{tr}\left((H-z)^{-1}-\left(H_{n, \theta}-z\right)^{-1}\right)=\frac{d}{d z} \ln (1+\theta g(z, n))=\int_{\mathbb{R}} \frac{\xi_{\theta}(\lambda, n)}{(\lambda-z)^{2}} d \lambda,
$$

where

$$
1+\theta g(z, n)=\exp \left(\int_{\mathbb{R}} \frac{\xi_{\theta}(\lambda, n)}{\lambda-z} d \lambda\right), \quad \xi_{\theta}(\lambda, n)=\frac{1}{\pi} \lim _{\varepsilon \downarrow 0} \arg (1+\theta g(\lambda+\mathrm{i} \varepsilon, n)) .
$$

By Theorem A.2 (iii) all moments of $\xi_{\theta}(\lambda, n) d \lambda$ are finite and $\int_{\mathbb{R}} \xi_{\theta}(\lambda, n) d \lambda=\theta$.

Taking logarithms in (5.15) and expanding yields as before 
Theorem 5.2. Let $\xi_{\theta}(\lambda, n)$ be defined as above. Then we have

$$
b_{\theta}^{(\ell)}(n)=(\ell+1) \int_{\mathbb{R}} \lambda^{\ell} \xi_{\theta}(\lambda, n) d \lambda,
$$

with

$$
b_{\theta}^{(0)}(n)=\theta, \quad b_{\theta}^{(\ell)}(n)=\theta(\ell+1) g_{\ell}(n)+\theta \sum_{j=1}^{\ell} g_{\ell-j}(n) b_{\theta}^{(j-1)}(n), \quad \ell \in \mathbb{N} .
$$

Again, in the special case $\ell=1$ we obtain

$$
b(n)=\frac{1}{\theta} \int_{\mathbb{R}} \lambda \xi_{\theta}(\lambda, n) d \lambda-\frac{\theta}{2} .
$$

In addition, we remark that letting the coupling constant $\theta$ tend to $\infty$ implies $H_{n, \theta} \rightarrow H_{n}^{\infty}$ in a suitable sense (i.e., norm resolvent sense on $\left\{f \in \ell^{2}(\mathbb{Z}) \mid\left\langle\delta_{n}, f\right\rangle=\right.$ $0\}$, cf. [19]). Similarly, $H_{n_{0}}^{\beta}$ can be obtained as the limit of the operator $H+$ $\theta\left\langle\delta_{n}^{\beta},.\right\rangle \delta_{n}^{\beta}$ as $\theta \rightarrow \infty$.

Clearly, the same procedure can be applied to (cf. Theorem A.2 (i), (iii))

$$
\gamma^{\beta}(z, n)=-\frac{\beta}{a(n)} \exp \left(\int_{\mathbb{R}} \frac{\xi^{\beta}(\lambda, n) d \lambda}{\lambda-z}\right), \quad z \in \mathbb{C} \backslash \sigma\left(H_{n}^{\beta}\right), \beta \in \mathbb{R} \backslash\{0\},
$$

where

$$
\xi^{\beta}(\lambda, n)=\frac{1}{\pi} \lim _{\varepsilon \downarrow 0} \arg \left(\gamma^{\beta}(\lambda+\mathrm{i} \varepsilon, n)\right)-\delta^{\beta}, \quad \delta^{\beta}= \begin{cases}0, & \beta a(n)<0 \\ 1, & \beta a(n)>0\end{cases}
$$

and $0 \leq \operatorname{sgn}(-a(n) \beta) \xi^{\beta}(\lambda, n) \leq 1$. This yields as before

Theorem 5.3. Let $\xi^{\beta}(\lambda, n)$ be defined as above. Then we have

$$
b^{\beta,(\ell)}(n)=(\ell+1) \frac{\beta}{a(n)} \int_{\mathbb{R}} \lambda^{\ell} \xi^{\beta}(\lambda, n) d \lambda, \quad \ell \in \mathbb{N}
$$

where

$$
\begin{aligned}
& b^{\beta,(0)}(n)=1+\beta^{2}, \\
& b^{\beta,(\ell)}(n)=(\ell+1) \gamma_{\ell}^{\beta}(n)-\frac{\beta}{a(n)} \sum_{j=1}^{\ell} \gamma_{\ell-j}^{\beta}(n) b^{\beta,(j-1)}(n), \quad \ell \in \mathbb{N} .
\end{aligned}
$$

Again specializing for $\ell=0$ in 5.21 we obtain

$$
a(n)=\frac{1}{\beta+\beta^{-1}} \int_{\mathbb{R}} \xi^{\beta}(\lambda, n) d \lambda .
$$

Finally, we want to find out when $\xi^{\beta_{j}}\left(\lambda, n_{0}\right), j=1,2$, for one fixed $n_{0}$ determines $a(n), b(n), n \in \mathbb{Z}$. Since $\xi^{\beta}\left(., n_{0}\right), \beta \in \mathbb{R}$ and $a\left(n_{0}\right)$ determines $\gamma^{\beta}\left(z, n_{0}\right)$ by 5.19 ) we conclude from Theorem 4.4

Corollary 5.4. Let $\beta_{1,2} \in \mathbb{R} \cup\{\infty\}$ be given. Then $\left(\beta_{j}, \xi^{\beta_{j}}\left(., n_{0}\right)\right), j=1,2$, and $a\left(n_{0}\right)$ for one fixed $n_{0} \in \mathbb{Z}$ uniquely determines $a(n)^{2}, b(n)$ for all $n \in \mathbb{Z}$. 


\section{REFLECTIONLESS OPERATORS}

Reflectionless operators have attracted a considerable amount of interest recently in connection with inverse spectral theory [2, 22, [35, 36] and completely integrable lattices [7, 32. In this section we show that the trace formulas of the previous section become particularly transparent in this case.

We will assume that $H$ is a bounded self-adjoint Jacobi operator. Hence its spectrum can be written as the complement of a countable union of disjoint open intervals, that is,

$$
\sigma(H)=\mathbb{R} \backslash \bigcup_{j \in J_{0} \cup\{\infty\}} \rho_{j},
$$

where $J \subseteq \mathbb{N}, J_{0}=J \cup\{0\}$,

$$
\begin{array}{cl}
\rho_{0}=\left(-\infty, E_{0}\right), & \rho_{\infty}=\left(E_{\infty}, \infty\right), \\
E_{0} \leq E_{2 j-1}<E_{2 j} \leq E_{\infty}, \quad & \rho_{j}=\left(E_{2 j-1}, E_{2 j}\right), \quad j \in J, \\
-\infty<E_{0}<E_{\infty}<\infty, & \rho_{j} \cap \rho_{k}=\emptyset \text { for } j \neq k .
\end{array}
$$

In addition, we will require that $H$ is reflectionless, that is, for all $n \in \mathbb{Z}$,

$$
\xi(\lambda, n)=\frac{1}{2} \text { for a.e. } \lambda \in \sigma_{e s s}(H) .
$$

By [22, Lemma 3.3 the requirement 6.3$]$ is equivalent to one of the following

(i). For some $n_{0} \in \mathbb{Z}, n_{1} \in \mathbb{Z} \backslash\left\{n_{0}, n_{0}+1\right\}$,

$$
\xi\left(\lambda, n_{0}\right)=\xi\left(\lambda, n_{0}+1\right)=\xi\left(\lambda, n_{1}\right)=\frac{1}{2} \text { for a.e } \lambda \in \sigma_{e s s}(H) .
$$

(ii). For some $n_{0} \in \mathbb{Z}$,

$$
\tilde{m}_{+}\left(\lambda+\mathrm{i} 0, n_{0}\right)=\overline{\tilde{m}_{-}\left(\lambda+\mathrm{i} 0, n_{0}\right)} \text { for a.e. } \lambda \in \sigma_{e s s}(H),
$$

where $\tilde{m}_{-}\left(\lambda+\mathrm{i} 0, n_{0}\right)$ abbreviates $\lim _{\varepsilon \downarrow 0} \tilde{m}_{-}\left(\lambda+\mathrm{i} \varepsilon, n_{0}\right)$.

The last equation implies

$$
u_{+}(\lambda+\mathrm{i} 0, n)=\overline{u_{-}(\lambda+\mathrm{i} 0, n)} \text { for a.e. } \lambda \in \sigma_{e s s}(H)
$$

for $u_{ \pm}(z, n)=c\left(z, n, n_{0}\right)+a\left(n_{0}\right) \tilde{m}_{ \pm}\left(z, n_{0}\right) s\left(z, n, n_{0}\right)$, where $c, s$ are the solutions of $\tau u=z u$ corresponding to the initial conditions $c\left(z, n_{0}, n_{0}\right)=s\left(z, n_{0}+1, n_{0}\right)=1$, $s\left(z, n_{0}, n_{0}\right)=c\left(z, n_{0}+1, n_{0}\right)=0$.

The name reflectionless will become clear in the next section. There the above conditions will turn out to be equivalent to the vanishing of the reflection coefficients $R_{ \pm}(z)$ (cf. 7.16$)$ ). For instance periodic operators, operators with purely discrete spectrum, and stationary solutions of the Toda hierarchy are special cases of reflectionless operators.

Next we turn to Dirichlet eigenvalues associated with $\tau$ corresponding to a Dirichlet boundary condition at $n \in \mathbb{Z}$. Associated with each spectral gap $\rho_{j}$ we set

$$
\mu_{j}(n)=\sup \left\{E_{2 j-1}\right\} \cup\left\{\lambda \in \rho_{j} \mid g(\lambda, n)<0\right\} \in \overline{\rho_{j}}, \quad j \in J .
$$

The numbers $\mu_{j}(n)$ are called Dirichlet eigenvalues of $H$ since we have

$$
\sigma\left(H_{n}^{\infty}\right)=\sigma_{\text {ess }}(H) \cup\left\{\mu_{j}(n)\right\}_{j \in J} .
$$

However, we want to point out that $\mu_{j}(n)$ is not necessarily an eigenvalue of $H_{n}^{\infty}$ unless $\mu_{j}(n) \notin \sigma_{\text {ess }}(H)$. 
The strict monotonicity of $g(\lambda, n)$ with respect to $\lambda \in \rho_{j}$, that is,

$$
\frac{d}{d \lambda} g(\lambda, n)=\left\langle\delta_{n},(H-\lambda)^{-2} \delta_{n}\right\rangle=\sum_{m \in \mathbb{Z}} G(\lambda, n, m)^{2}>0, \quad \lambda \in \rho_{j},
$$

then yields

$$
\begin{array}{ll}
g(\lambda, n)<0, & \lambda \in\left(E_{2 j-1}, \mu_{j}(n)\right), \\
g(\lambda, n)>0, & \lambda \in\left(\mu_{j}(n), E_{2 j}\right),
\end{array} \quad j \in J .
$$

Thus we conclude $\xi(\lambda, n)=1, \lambda \in\left(E_{2 j-1}, \mu_{j}(n)\right)$ and $\xi(\lambda, n)=0, \lambda \in\left(\mu_{j}(n), E_{2 j}\right)$, $j \in J$. Using this information to evaluate the exponential Herglotz representation of $g(z, n)$ then implies ([22], Lemma 1.1)

$$
g(z, n)=\frac{-1}{\sqrt{z-E_{0}} \sqrt{z-E_{\infty}}} \prod_{j \in J} \frac{z-\mu_{j}(n)}{\sqrt{z-E_{2 j-1}} \sqrt{z-E_{2 j}}},
$$

where the square root branch used is defined as $\sqrt{z}=|\sqrt{z}| \exp (\mathrm{i} \arg (z) / 2),-\pi<$ $z \leq \pi$. In addition, denoting by $\chi_{\Omega}($.$) the characteristic function of the set \Omega \subset \mathbb{R}$, one can represent $\xi(\lambda, n)$ by

$$
\begin{aligned}
\xi(\lambda, n)= & \frac{1}{2}\left(\chi_{\left(E_{0}, \infty\right)}(\lambda)+\chi_{\left(E_{\infty}, \infty\right)}(\lambda)\right) \\
& +\frac{1}{2} \sum_{j \in J}\left(\chi_{\left(E_{2 j-1}, \infty\right)}(\lambda)+\chi_{\left(E_{2 j}, \infty\right)}(\lambda)-2 \chi_{\left(\mu_{j}(n), \infty\right)}(\lambda)\right) \\
= & \frac{1}{2} \chi_{\left(E_{0}, E_{\infty}\right)}(\lambda)+\frac{1}{2} \sum_{j \in J}\left(\chi_{\left(E_{2 j-1}, \mu_{j}(n)\right)}(\lambda)-\chi_{\left(\mu_{j}(n), E_{2 j}\right)}(\lambda)\right) \\
& +\chi_{\left(E_{\infty}, \infty\right)}(\lambda) \text { for a.e. } \lambda \in \mathbb{R} .
\end{aligned}
$$

Evaluation of 5.6 shows

$$
b^{(\ell)}(n)=\frac{1}{2}\left(E_{0}^{\ell}+E_{\infty}^{\ell}+\sum_{j \in J}\left(E_{2 j-1}^{\ell}+E_{2 j}^{\ell}-2 \mu_{j}(n)^{\ell}\right)\right)
$$

and in the special case $\ell=1$

$$
b(n)=\frac{1}{2}\left(E_{0}+E_{\infty}+\sum_{j \in J}\left(E_{2 j-1}+E_{2 j}-2 \mu_{j}(n)\right)\right) .
$$

The formulas for $\ell=1,2$ were first given in [2], Theorem 5.2.

Next, we want to address the problem of expressing $a(n)^{2}$ as a function of $E_{j}$ and $\mu_{j}(n)$. This endeavor turns out to be impossible unless we introduce additional data. This will be done first by defining

$$
\left\{\tilde{\mu}_{j}(n)\right\}_{j \in \tilde{J}}=\left\{\mu_{j}(n)\right\}_{j \in J} \cup \sigma_{p}\left(H_{n}^{\infty}\right), \quad \tilde{J} \subseteq \mathbb{N}
$$

and $\tilde{E}_{0}=E_{0}, \tilde{E}_{\infty}=E_{\infty}$,

$$
\tilde{E}_{2 j-1}=\sup \left\{E \in \sigma(H) \mid E<\tilde{\mu}_{j}(n)\right\}, \quad \tilde{E}_{2 j}=\inf \left\{E \in \sigma(H) \mid \tilde{\mu}_{j}(n)<E\right\} .
$$

A few remarks are in order:

Remark 6.1. (i). We note that $\tilde{\mu}_{j}=\mu_{k}$ implies $\tilde{E}_{2 j-1}=E_{2 k-1}, \tilde{E}_{2 j}=E_{2 k}$ and $\tilde{E}_{2 j-1}<\tilde{E}_{2 j}$ implies $\tilde{\mu}_{j}(n)=\mu_{k}(n)$ for some $k \in J$. Indeed, if $\tilde{E}_{2 j-1}<\tilde{E}_{2 j}$ we infer $\lim _{\lambda \rightarrow \tilde{\mu}_{j}(n), \lambda \in\left(\tilde{E}_{2 j-1}, \tilde{E}_{2 j}\right)} g(\lambda, n)=0$ and hence $\tilde{\mu}_{j}(n)=\mu_{k}(n)$ for some $k \in J$ by monotonicity of $g(., n)$ in spectral gaps. In other words, computing all previous 
formulas with $\mu_{j}(n), E_{j}$ replaced by $\tilde{\mu}_{j}(n), \tilde{E}_{j}$ leaves them unchanged since the new factors drop out.

(ii). Our notation concerning $\tilde{E}_{j}$ is imprecise since the list of numbers $\left[\tilde{E}_{j}\right]_{j \in \tilde{J}}$ might, in general, depend on $n$. Suppose for instance, that $\tilde{\mu}_{j}(n)$ is also an eigenvalue of $H$ such that $\tilde{E}_{2 j-1}=\tilde{\mu}_{j}(n)=\tilde{E}_{2 j}$. Then the pair $\tilde{E}_{2 j-1}, \tilde{E}_{2 j}$ shows up in the list corresponding to $n$ but not in the one corresponding to $n+1$ since the eigenfunction for $\tilde{\mu}_{j}(n)$ cannot vanish at two consecutive points.

Moreover, following [22], we introduce the numbers

$$
\tilde{R}_{j}(n)=\lim _{\varepsilon \downarrow 0} \mathrm{i} \varepsilon g\left(\tilde{\mu}_{j}(n)+\mathrm{i} \varepsilon, n\right)^{-1} \geq 0,
$$

and

$$
\tilde{\sigma}_{j}(n)=\left\{\begin{array}{cc}
\lim _{\varepsilon \downarrow 0} h\left(\tilde{\mu}_{j}(n)+\mathrm{i} \varepsilon, n\right) & \text { if } \tilde{R}_{j}(n)>0 \\
2 & \text { if } \tilde{R}_{j}(n)=0
\end{array} .\right.
$$

The actual value of $\tilde{\sigma}_{j}(n)$ if $\tilde{R}_{j}(n)=0$ is immaterial and is chosen in accordance with [22. The above limits exist if $\tilde{\mu}_{j} \in \sigma\left(H_{n}^{\infty}\right)$ (i.e., if $\tilde{R}_{j}(n)>0$ ) and $\tilde{\sigma}_{j}(n)$ is either \pm 1 (depending on whether $\tilde{\mu}_{j}$ is an eigenvalue of $H_{ \pm, n}$ ) or in $\left(-1,+1\right.$ ) (if $\tilde{\mu}_{j}$ is an eigenvalue of both $H_{ \pm, n}$ and hence also of $H$ ). For more details see [22].

The numbers $\tilde{R}_{j}(n)$ can be evaluated using 6.9

$$
\tilde{R}_{j}(n)=\frac{\sqrt{\tilde{\mu}_{j}(n)-E_{0}} \sqrt{\tilde{\mu}_{j}(n)-E_{\infty}} \sqrt{\tilde{\mu}_{j}(n)-E_{2 j-1}} \sqrt{\tilde{\mu}_{j}(n)-E_{2 j}}}{\prod_{k \in J \backslash\{j\}} \frac{\tilde{\mu}_{j}(n)-\mu_{k}(n)}{\sqrt{\tilde{\mu}_{j}(n)-E_{2 k-1}} \sqrt{\tilde{\mu}_{j}(n)-E_{2 k}}}} .
$$

If $\tilde{\mu}_{j}=\mu_{k}=E_{2 k}=E_{2 j-1}$ for some $k$ (resp. $\tilde{\mu}_{j}=\mu_{k}=E_{2 k-1}=E_{2 j}$ ) the vanishing factors $\tilde{\mu}_{j}-\mu_{k}$ in the denominator and $\tilde{\mu}_{j}-E_{2 j}$ (resp. $\tilde{\mu}_{j}-E_{2 j-1}$ ) in the numerator have to be omitted. In particular, we want to point out that $\tilde{R}_{j}(n)$ depend on $E_{j}, \mu_{j}$ only.

In addition, we require that the singularly continuous spectrum of $H_{n}^{\infty}$ is empty (the absolutely continuous spectrum being taken care of by the reflectionless condition). Then it is shown in [22] that the spectral data $E_{j}, j \in J \cup\{0, \infty\}$ plus $\mu_{j}\left(n_{0}\right)$, $j \in J$ plus $\tilde{\sigma}_{j}\left(n_{0}\right), j \in \tilde{J}$ for one fixed $n_{0} \in \mathbb{Z}$ are minimal and uniquely determine $a(n)^{2}, b(n)$. (To be precise, the class of operators considered here is slightly larger than the one in 22], however, the same proof applies.) Moreover, necessary and sufficient conditions for given spectral data to be the spectral data of some Jacobi operator were derived. Here we want to focus on the reconstruction of $a(n)^{2}, b(n)$ from given spectral data as above and present an explicit expression of $a(n)^{2}, b(n)$ in terms of the spectral data.

Our point of departure will be the formulas (use 4.15) and (4.17))

$$
\begin{aligned}
a(n)^{2} m_{+}(z, n) \pm a(n-1)^{2} m_{-}(z, n) & =\mp z \pm b(n)-\left\{\begin{array}{l}
\frac{1}{g(z, n)} \\
\frac{h(z, n)}{g(z, n)}
\end{array}\right. \\
& =-\sum_{j=0}^{\infty} \frac{c_{ \pm, j}(n)}{z^{j+1}}
\end{aligned}
$$


where the coefficients $c_{ \pm, j}(n)$ are to be determined. Arguing similarly as for 1.4 one obtains

$$
c_{ \pm, \ell}(n)=\int_{\mathbb{R}} \lambda^{\ell}\left(a(n)^{2} d \rho_{+, n}(\lambda) \pm a(n-1)^{2} d \rho_{-, n}(\lambda)\right), \quad \ell \in \mathbb{N}_{0},
$$

where $d \rho_{ \pm, n}(\lambda)$ are the spectral measures of $H_{ \pm, n}$ associated with the vector $\delta_{n \pm 1}$.

The evaluation of this integral will now be done for the minus sign. Due to the reflectionless condition, the integral over the (absolutely) continuous spectrum is zero (there is no singularly continuous part by assumption) and it remains to evaluate the pure point part. To do this it suffices to know the jumps of the measure which are given by the residues of the corresponding Herglotz function. Evaluating the residues (using (6.18) plus the notation from above) shows

$$
c_{-, \ell}(n)=\sum_{j \in \tilde{J}} \tilde{\sigma}_{j}(n) \tilde{R}_{j}(n) \tilde{\mu}_{j}(n)^{\ell}, \quad \ell \in \mathbb{N}_{0}
$$

Clearly it suffices to sum over all $\tilde{\mu}_{j}(n) \in \sigma_{p}\left(H_{n}^{\infty}\right)$ since for all other terms we have $\tilde{R}_{j}(n)=0$.

Next we turn to the coefficients $c_{+, \ell}(n)$. They can be determined from (cf. (5.5))

$$
\frac{1}{g(z, n)}=-z \exp \left(-\sum_{\ell=1}^{\infty} \frac{b^{(\ell)}(n)}{\ell z^{\ell}}\right)
$$

which implies

$$
\begin{aligned}
c_{+,-2}(n) & =1, \\
c_{+, \ell-2}(n) & =\frac{1}{\ell} \sum_{j=1}^{\ell} c_{+, \ell-j-2}(n) b^{(j)}(n), \quad \ell \in \mathbb{N} .
\end{aligned}
$$

Thus $c_{+, \ell}(n)$ are expressed in terms of $E_{j}, \mu_{j}(n)$. Here $c_{+,-2}(n)$ and $c_{+,-1}(n)$ have been introduced for notational convenience only.

In particular, combining the case $\ell=0$ with our previous results we obtain

$$
a\left(n-{ }_{1}^{0}\right)^{2}=\frac{b^{(2)}(n)-b(n)}{4} \pm \sum_{j \in \tilde{J}} \frac{\tilde{\sigma}_{j}(n)}{2} \tilde{R}_{j}(n) .
$$

Similarly, for $\ell=1$,

$$
\begin{aligned}
b(n \pm 1)= & \frac{1}{a\left(n-{ }_{1}^{0}\right)^{2}}\left(\frac{2 b^{(3)}(n)-3 b(n) b^{(2)}(n)+b(n)^{3}}{12}\right. \\
& \left. \pm \sum_{j \in \tilde{J}} \frac{\tilde{\sigma}_{j}(n)}{2} \tilde{R}_{j}(n) \tilde{\mu}_{j}(n)\right) .
\end{aligned}
$$

However, these formulas are only the tip of the iceberg. Combining

$$
c_{ \pm, \ell}(n)=a(n)^{2} m_{+, \ell}(n) \pm a(n-1)^{2} m_{-, \ell}(n)
$$

with some basic facts from the moment problem we obtain our main result:

Theorem 6.2. Let $H$ be a given bounded reflectionless Jacobi operator. Suppose the singularly continuous spectrum of $H_{n}^{\infty}$ is empty and the spectral data corresponding 
to $H$ (as above) are given for one fixed $n \in \mathbb{Z}$. Then the sequences $a^{2}, b$ can be expressed explicitly in terms of the spectral data as follows

$$
\begin{aligned}
a\left(n \pm k-{ }_{0}^{0}\right)^{2} & =\frac{C_{ \pm, n}(k+1) C_{ \pm, n}(k-1)}{C_{ \pm, n}(k)^{2}}, \\
b(n \pm k) & =\frac{D_{ \pm, n}(k)}{C_{ \pm, n}(k)}-\frac{D_{ \pm, n}(k-1)}{C_{ \pm, n}(k-1)}, \quad k \in \mathbb{N},
\end{aligned}
$$

where $C_{ \pm, n}(0)=1, D_{ \pm, n}(0)=0$,

$$
D_{ \pm, n}(k)=\operatorname{det}\left(\begin{array}{ccccc}
m_{ \pm, 0}(n) & m_{ \pm, 1}(n) & \cdots & m_{ \pm, k-2}(n) & m_{ \pm, k}(n) \\
m_{ \pm, 1}(n) & m_{ \pm, 2}(n) & \cdots & m_{ \pm, k-1}(n) & m_{ \pm, k+1}(n) \\
\vdots & \vdots & \ddots & \vdots & \vdots \\
m_{ \pm, k-1}(n) & m_{ \pm, k}(n) & \cdots & m_{ \pm, 2 k-3}(n) & m_{ \pm, 2 k-1}(n)
\end{array}\right) \text {, }
$$

and $m_{ \pm, \ell}(n)=\frac{c_{+, \ell}(n) \pm c_{-, \ell}(n)}{2 a\left(n-{ }_{1}^{0}\right)}$. The quantities $a(n)^{2}, a(n-1)^{2}$, and $c_{ \pm, \ell}(n)$ have to be expressed in terms of the spectral data using (6.23), 6.22, 6.20) and (6.11).

Proof. It remains to show the expressions $(6.26)$ and $(6.27)$ for $a(n)$ and $b(n)$ in terms of the moments $M_{ \pm, \ell}\left(n_{0}\right), \ell \in \mathbb{N}$. Both can be found in [1] (first equation on page 5). However, the equation for $b(n)$ here differs from the one in 1 since we have performed the integration (see [38, Section 2.5 for details).

In the special case of periodic Jacobi operators, the formula 6.23 was first given in [7. In addition, we get a discrete version of Borg's theorem.

Corollary 6.3. Let $H$ be a reflectionless Jacobi operator with spectrum consisting of only one band, that is $\sigma(H)=\left[E_{0}, E_{\infty}\right]$. Then the sequences $a(n)^{2}, b(n)$ are necessarily constant

$$
a(n)^{2}=\frac{\left(E_{\infty}-E_{0}\right)^{2}}{16}, \quad b(n)=\frac{E_{0}+E_{\infty}}{2} .
$$

The special case where $H$ is periodic seems due to [12] (Proposition 2 on p. 451). The formula for $b(n)$ also follows directly from 5.12 .

Remark 6.4. (i). If $J$ is finite, that is, $H$ has only finitely many spectral gaps, then $\left\{\tilde{\mu}_{j}(n)\right\}_{j \in \tilde{J}}=\left\{\mu_{j}(n)\right\}_{j \in J}$ and we can forget about the additional $\mu$ 's.

(ii). The reader might wonder whether a similar procedure for one-dimensional Schrödinger operators $H=-\frac{d^{2}}{d x^{2}}+V(x)$ is possible. This is in fact the case but under more restrictive conditions on $V(x)$. Without going into technical details we remark that in the continuous case the asymptotic expansions of the Weyl mfunctions contain the information of all derivatives of $V$ at the base point. Hence if $V$ is assumed real analytic (e.g., finite gap) it can be expressed in terms of its derivatives using Taylor's formula.

(iii). Concerning general Jacobi operators we note that Theorem 4.4 indicates that $a\left(n_{0}\right)^{2}, \gamma_{\ell}^{\beta_{j}}\left(n_{0}\right), j=1,2, \ell \in \mathbb{N}$ is solvable for $a(n)^{2}, b(n)$ as well. 
Finally, we turn to general eigenvalues associated with $H_{n}^{\beta}$. Associated with each spectral gap $\rho_{j}$ we set

$$
\lambda_{j}^{\beta}(n)=\sup \left\{E_{2 j-1}\right\} \cup\left\{\lambda \in \rho_{j} \mid \gamma^{\beta}(\lambda, n)<0\right\} \in \overline{\rho_{j}}, \quad j \in J .
$$
is,

The strict monotonicity of $\gamma^{\beta}(\lambda, n)$ with respect to $\lambda \in \rho_{j}, j \in J_{0} \cup\{\infty\}$, that

$$
\frac{d}{d \lambda} \gamma^{\beta}(\lambda, n)=\left(1+\beta^{2}\right)\left\langle\delta_{n}^{\beta},(H-\lambda)^{-2} \delta_{n}^{\beta}\right\rangle, \quad \lambda \in \rho_{j},
$$

then yields

$$
\begin{array}{ll}
\gamma^{\beta}(\lambda, n)<0, & \lambda \in\left(E_{2 j-1}, \lambda_{j}^{\beta}(n)\right), \\
\gamma^{\beta}(\lambda, n)>0, & \lambda \in\left(\lambda_{j}^{\beta}(n), E_{2 j}\right),
\end{array} \quad j \in J .
$$

Since $\gamma^{\beta}(\lambda, n)$ is positive (resp. negative) for $a(n) \beta>0$ (resp. $\left.a(n) \beta<0\right)$ as $\lambda \rightarrow \infty$ (resp. $\lambda \rightarrow-\infty)$, there must be an additional zero $\lambda_{\infty}^{\beta}$ for $\lambda \geq E_{\infty}$ (resp. $\lambda \leq E_{0}$ ). Summarizing, $\xi^{\beta}(\lambda, n)$ is given by

$$
\begin{aligned}
\xi^{\beta}(\lambda, n)= & \frac{1}{2} \chi_{\left(E_{0}, E_{\infty}\right)}(\lambda)+\frac{1}{2} \sum_{j \in J}\left(\chi_{\left(E_{2 j-1}, \lambda_{j}^{\beta}(n)\right)}(\lambda)-\chi_{\left(\lambda_{j}^{\beta}(n), E_{2 j}\right)}(\lambda)\right) \\
& +\chi_{\left(E_{\infty}, \lambda_{\infty}^{\beta}\right)}(\lambda), \quad a(n) \beta>0
\end{aligned}
$$

and

$$
\begin{aligned}
\xi^{\beta}(\lambda, n)= & -\frac{1}{2} \chi_{\left(E_{0}, E_{\infty}\right)}(\lambda)+\frac{1}{2} \sum_{j \in J}\left(\chi_{\left(E_{2 j-1}, \lambda_{j}^{\beta}(n)\right)}(\lambda)-\chi_{\left(\lambda_{j}^{\beta}(n), E_{2 j}\right)}(\lambda)\right) \\
& -\chi_{\left(\lambda_{\infty}^{\beta}, E_{0}\right)}(\lambda), \quad a(n) \beta<0 .
\end{aligned}
$$

Thus we have for $\beta \neq 0, \infty$,

$$
\gamma^{\beta}(z, n)=\frac{z-\lambda_{\infty}^{\beta}(n)}{\sqrt{z-E_{0}} \sqrt{z-E_{\infty}}} \prod_{j \in J} \frac{z-\lambda_{j}^{\beta}(n)}{\sqrt{z-E_{2 j-1}} \sqrt{z-E_{2 j}}},
$$

and we remark that the numbers $\lambda_{j}^{\beta}(n)$ are related to the spectrum of $H_{n}^{\beta}$ as follows

$$
\sigma\left(H_{n}^{\beta}\right)=\sigma_{e s s}(H) \cup\left\{\lambda_{j}^{\beta}(n)\right\}_{j \in J \cup\{\infty\}} .
$$

Again we point out that $\lambda_{j}^{\beta}(n)$ is not necessarily an eigenvalue of $H_{n}^{\beta}$ unless $\lambda_{j}^{\beta}(n) \notin$ $\sigma_{\text {ess }}(H)$.

Evaluation of (5.6) shows

$$
\begin{aligned}
b^{\beta,(\ell)}(n)= & \frac{-\beta}{2 a(n)}\left(E_{0}^{\ell+1}+E_{\infty}^{\ell+1}-2 \lambda_{\infty}^{\beta}(n)^{\ell+1}\right. \\
& \left.+\sum_{j \in J}\left(E_{2 j-1}^{\ell+1}+E_{2 j}^{\ell+1}-2 \lambda_{j}^{\beta}(n)^{\ell+1}\right)\right)
\end{aligned}
$$

and in the special case $\ell=0$,

$$
a(n)=\frac{1}{2\left(\beta+\beta^{-1}\right)}\left(E_{0}+E_{\infty}-2 \lambda_{\infty}^{\beta}(n)+\sum_{j \in J}\left(E_{2 j-1}+E_{2 j}-2 \lambda_{j}^{\beta}(n)\right)\right) .
$$




\section{SCATtering THEORY}

One important class of Jacobi operators are periodic ones. In this section we want to consider scattering theory with periodic background operators and apply the results of Section 5. Even though this problem arises naturally if one considers an infinite harmonic crystal (with $N$ atoms in the base cell) with impurities, not too many articles are available on this problem (cf., e.g., [15], 28]). The case with constant background (i.e., only one atom in the base cell) is treated, for instance in 9], 27]. For a comprehensive treatment in the case of Schrödinger operators with fairly arbitrary backgrounds we refer the reader to [23] and the references therein.

We first recall some basic facts from the theory of periodic operators (cf., e.g., 7], Appendix B, 30, 32]). Let $H_{p}$ be a Jacobi operator associated with periodic sequences $a_{p} \neq 0, b_{p}$, that is,

$$
a_{p}(n+N)=a_{p}(n), \quad b_{p}(n+N)=b_{p}(n),
$$

for some fixed $N \in \mathbb{N}$. The spectrum of $H_{p}$ is purely absolutely continuous and consists of a finite number of gaps, that is,

$$
\sigma(H)=\bigcup_{j=1}^{N}\left[E_{p, 2 j-2}, E_{p, 2 j-1}\right], \quad E_{p, 0}<\cdots<E_{p, 2 N-1}
$$

Moreover, Floquet theory implies the existence of solutions $u_{p, \pm}(z,$.$) of \tau_{p} u=z u$, $z \in \mathbb{C}\left(\tau_{p}\right.$ the difference expression corresponding to $\left.H_{p}\right)$ satisfying

$$
u_{p, \pm}(z, n+N)=m^{ \pm}(z) u_{p, \pm}(z, n)
$$

and hence

$$
u_{p, \pm}(z, n)=p_{ \pm}(z, n) \exp ( \pm \mathrm{i} q(z) n), \quad p_{ \pm}(z, n)=p_{ \pm}(z, n+N)
$$

where $m^{ \pm}(z)=\exp ( \pm \mathrm{i} q(z) N) \in \mathbb{C}$ are called Floquet multipliers and $q(z)$ is called Floquet momentum $\left(m^{ \pm}(z)\right.$ is not related to the Weyl $m$-function $\left.m_{ \pm}(z, n)\right)$. $m^{ \pm}(z)$ satisfy $m^{+}(z) m^{-}(z)=1, m^{ \pm}(z)^{2}=1$ for $z \in\left\{E_{p, j}\right\}_{j=0}^{2 N-1},\left|m^{ \pm}(z)\right|=1$ for $z \in \sigma\left(H_{p}\right)$, and $\left|m^{+}(z)\right|<1$ for $z \in \mathbb{C} \backslash \sigma\left(H_{p}\right)$. (This says in particular, that $u_{p, \pm}(z,$.$) are bounded for z \in \sigma\left(H_{p}\right)$ and linearly independent for $z \in \mathbb{C} \backslash\left\{E_{j}\right\}_{j=0}^{2 N-1}$.) Requiring $m^{ \pm}(\lambda)=\lim _{\varepsilon \downarrow 0} m^{ \pm}(\lambda+\mathrm{i} \varepsilon), \lambda \in \sigma\left(H_{p}\right)$ determines $m^{ \pm}(z)$ uniquely.

We are going to investigate scattering theory for the pair $\left(H, H_{p}\right)$, where $H$ is a Jacobi operator satisfying

$$
\sum_{n \in \mathbb{Z}}\left|n\left(a(n)-a_{p}(n)\right)\right|<\infty, \quad \sum_{n \in \mathbb{Z}}\left|n\left(b(n)-b_{p}(n)\right)\right|<\infty .
$$

By 37, Theorem 5.1 the requirement (7.5) implies that the essential spectrum of $H$ is equal to $\sigma\left(H_{p}\right)$ and purely absolutely continuous. Moreover, the point spectrum of $H$ is finite and confined to the spectral gaps of $H_{p}$, that is, $\sigma_{p}(H) \subset \mathbb{R} \backslash \sigma\left(H_{p}\right)$.

As in the proof of [37, Theorem 5.1 one can use the sum equation

$$
u_{ \pm}(z, n)=\frac{a_{p}\left(n-\begin{array}{l}
0 \\
1
\end{array}\right)}{a\left(n-{ }_{1}^{0}\right)} u_{p, \pm}(z, n) \mp \sum_{m=-\infty}^{\substack{n+1 \\
n-1}} \frac{a_{p}\left(n-\begin{array}{l}
0 \\
1
\end{array}\right)}{a\left(n-\begin{array}{l}
0 \\
1
\end{array}\right)} K(z, n, m) u_{ \pm}(z, m)
$$


where

$$
\begin{aligned}
K(z, n, m)= & \frac{\left(\left(\tau-\tau_{p}\right) u_{p,-}(z)\right)(m) u_{p,+}(z, n)-u_{p,-}(z, n)\left(\left(\tau-\tau_{p}\right) u_{p,+}(z)\right)(m)}{W_{p}\left(u_{p,-}(z), u_{p,+}(z)\right)} \\
= & \frac{s_{p}(\lambda, n, m+1)}{a_{p}(m+1)}\left(a(m)-a_{p}(m)\right)+\frac{s_{p}(\lambda, n, m)}{a_{p}(m)}\left(b(m)-b_{p}(m)\right) \\
& +\frac{s_{p}(\lambda, n, m-1)}{a_{p}(m-1)}\left(a(m-1)-a_{p}(m-1)\right)
\end{aligned}
$$

$\left(W_{p}(., .\right.$.$) denotes the Wronskian formed with a_{p}$ rather than $\left.a\right)$ to show the existence of solutions $u_{ \pm}(z,$.$) of \tau u=z u$ satisfying

$$
\lim _{n \rightarrow \pm \infty} \exp (\mp \operatorname{Im}(q(z)) n)\left|u_{ \pm}(z, n)-u_{p, \pm}(z, n)\right|=0, \quad z \in \mathbb{C} .
$$

Since we are most of the time interested in the case $z \in \sigma\left(H_{p}\right)$ we shall normalize $u_{p, \pm}(\lambda, 0)=1$ for $\lambda \in \sigma\left(H_{p}\right)$. In what follows we will freely use the notation and results found in [7, Appendix B. In particular, note that we have $\overline{u_{p, \pm}(\lambda)}=u_{p, \mp}(\lambda)$, where the bar denotes complex conjugation. Since one computes

$$
W\left(u_{ \pm}(\lambda), \overline{u_{ \pm}(\lambda)}\right)=W_{p}\left(u_{p, \pm}(\lambda), u_{p, \mp}(\lambda)\right)=\mp \frac{2 \mathrm{i} \sin (q(\lambda) N)}{s_{p}(\lambda, N)}, \quad \lambda \in \sigma\left(H_{p}\right)
$$

$\left(s_{p}(\lambda, n)\right.$ is the solution of $\tau_{p} u=z u$ corresponding to the initial condition $s(\lambda, 0)=$ $\left.0, s_{p}(\lambda, 1)=1\right)$ we conclude that $u_{ \pm}(\lambda), \overline{u_{ \pm}(\lambda)}$ are linearly independent for $\lambda$ in the interior of $\sigma\left(H_{p}\right)$ (if two bands collide at $E$, numerator and denominator of 7.9 p both approach zero when $\lambda \rightarrow E$ and have a nonzero limit). Hence we might set

$$
u_{ \pm}(\lambda, n)=\alpha(\lambda) \overline{u_{\mp}(\lambda, n)}+\beta_{\mp}(\lambda) u_{\mp}(\lambda, n), \quad \lambda \in \sigma\left(H_{p}\right),
$$

where

$$
\begin{aligned}
\alpha(\lambda) & =\frac{W\left(u_{\mp}(\lambda), u_{ \pm}(\lambda)\right)}{W\left(u_{\mp}(\lambda), \overline{u_{\mp}(\lambda)}\right)}=\frac{s_{p}(\lambda, N)}{2 \mathrm{i} \sin (q(\lambda) N)} W\left(u_{-}(\lambda), u_{+}(\lambda)\right), \\
\beta_{ \pm}(z) & =\frac{W\left(u_{\mp}(\lambda)\right), \overline{u_{ \pm}(\lambda)}}{W\left(u_{ \pm}(\lambda), \overline{u_{ \pm}(\lambda)}\right)}= \pm \frac{s_{p}(\lambda, N)}{2 \sin (q(\lambda) N)} W\left(u_{\mp}(\lambda), \overline{u_{ \pm}(\lambda)}\right) .
\end{aligned}
$$

The function $\alpha(\lambda)$ can be defined for all $\lambda \in \mathbb{C} \backslash\left\{E_{p, j}\right\}$. Note that we have

$$
|\alpha(\lambda)|^{2}=1+\left|\beta_{ \pm}(\lambda)\right|^{2} \text { and } \overline{\beta_{ \pm}(\lambda)}=-\beta_{\mp}(\lambda) .
$$

Using (7.6) one can also show

$$
W\left(u_{-}(\lambda), u_{+}(\lambda)\right)=W_{p}\left(u_{p,-}(\lambda), u_{p,+}(\lambda)\right)+\sum_{n \in \mathbb{Z}} u_{ \pm}(\lambda, n)\left(\left(\tau-\tau_{p}\right) u_{p, \mp}(\lambda)\right)(n)
$$

and

$$
W\left(u_{\mp}(\lambda), \overline{u_{ \pm}(\lambda)}\right)=\mp \sum_{n \in \mathbb{Z}} u_{ \pm}(\lambda, n)\left(\left(\tau-\tau_{p}\right) u_{p, \pm}(\lambda)\right)(n) .
$$

We now define the scattering matrix

$$
S(\lambda)=\left(\begin{array}{cc}
T(\lambda) & R_{-}(\lambda) \\
R_{+}(\lambda) & T(\lambda)
\end{array}\right), \quad \lambda \in \sigma\left(H_{p}\right)
$$

of the pair $\left(H, H_{p}\right)$, where $T(\lambda)=\alpha(\lambda)^{-1}$ and $R_{ \pm}(\lambda)=\alpha(\lambda)^{-1} \beta_{ \pm}(\lambda)$. The matrix $S(\lambda)$ is easily seen to be unitary since by $(7.13)|T(\lambda)|^{2}+\left|R_{ \pm}(\lambda)\right|^{2}=1$ and $T(\lambda) \overline{R_{+}(\lambda)}=-\overline{T(\lambda)} R_{-}(\lambda)$. 
The quantities $T(\lambda)$ and $R_{ \pm}(\lambda)$ are called transmission and reflection coefficients respectively. The following equation further explains this notation:

$$
T(\lambda) u_{ \pm}(\lambda, n)=\left\{\begin{array}{ll}
T(\lambda) u_{p, \pm}(\lambda, n), & n \rightarrow \pm \infty \\
u_{p, \pm}(\lambda, n)+R_{\mp}(\lambda) u_{p, \mp}(\lambda, n), & n \rightarrow \mp \infty
\end{array}, \lambda \in \sigma\left(H_{p}\right)\right.
$$

Clearly 6.4 implies $R_{ \pm}(\lambda)=0$, explaining the term reflectionless in the previous section. The quantities $T(\lambda)$ and $R_{ \pm}(\lambda)$ can be expressed in terms of $\tilde{m}_{ \pm}(z)=$ $\tilde{m}_{ \pm}(z, 0)$ as follows

$$
\begin{aligned}
T(\lambda) & =\frac{\overline{u_{ \pm}(\lambda, 0)}}{\overline{u_{\mp}(\lambda, 0)}} \frac{2 \mathrm{i} \operatorname{Im}\left(\tilde{m}_{ \pm}(\lambda+\mathrm{i} 0)\right)}{\tilde{m}_{-}(\lambda+\mathrm{i} 0)+\tilde{m}_{+}(\lambda+\mathrm{i} 0)}, \\
R_{ \pm}(\lambda) & =-\overline{u_{ \pm}(\lambda, 0)} \frac{\tilde{m}_{\mp}(\lambda+\mathrm{i} 0)+\tilde{m}_{ \pm}(\lambda+\mathrm{i} 0)}{u_{ \pm}(\lambda, 0)}, \quad \lambda \in \sigma\left(H_{p}\right) .
\end{aligned}
$$

In addition, one verifies

$$
\begin{aligned}
g(\lambda+\mathrm{i} 0, n) & =\frac{u_{-}(\lambda, n) u_{+}(\lambda, n)}{W\left(u_{-}(\lambda), u_{+}(\lambda)\right)}=T(\lambda) \frac{s_{p}(\lambda, N)}{2 \mathrm{i} \sin (q(\lambda) N)} u_{-}(\lambda, n) u_{+}(\lambda, n) \\
& =\frac{s_{p}(\lambda, N)}{2 \mathrm{i} \sin (q(\lambda) N)}\left|u_{ \pm}(\lambda, n)\right|^{2}\left(1+R_{ \pm}(\lambda) \frac{u_{ \pm}(\lambda, n)}{\overline{u_{ \pm}(\lambda, n)}}\right), \quad \lambda \in \sigma\left(H_{p}\right) .
\end{aligned}
$$

Construct the list $\left(E_{j}\right)_{j=0}^{2 M+1}$ by taking all $E_{p, j}$ plus two copies of each eigenvalue of $H$. We can assume $E_{0} \leq E_{1}<E_{2} \leq \cdots<E_{2 M} \leq E_{2 M+1}$ and equality holds if and only if $E_{2 j}=E_{2 j+1}$ is an eigenvalue of $H$. Define the Dirichlet eigenvalues $\mu_{j}(n)$ associated with each spectral gap $\left(E_{2 j+1}, E_{2 j+2}\right)$ as in $(6.5)$. Then we infer

$$
\begin{aligned}
\xi(\lambda, n)= & \frac{1}{2} \chi_{\left(E_{0}, E_{\infty}\right)}(\lambda)+\frac{1}{2} \sum_{j=1}^{M}\left(\chi_{\left(E_{2 j-1}, \mu_{j}(n)\right)}(\lambda)-\chi_{\left(\mu_{j}(n), E_{2 j}\right)}(\lambda)\right) \\
& +\chi_{\left(E_{\infty}, \infty\right)}(\lambda)+\frac{1}{\pi} \arg \left(1+R_{ \pm}(\lambda) \frac{u_{ \pm}(\lambda, n)}{u_{ \pm}(\lambda, n)}\right) \chi_{\sigma\left(H_{p}\right)}(\lambda)
\end{aligned}
$$

since we have

$$
\xi(\lambda, n)=\frac{1}{2}+\frac{1}{\pi} \arg \left(1+R_{ \pm}(\lambda) \frac{u_{ \pm}(\lambda, n)}{\overline{u_{ \pm}(\lambda, n)}}\right), \lambda \in \sigma\left(H_{p}\right) .
$$

Hence we obtain from 5.6

$$
\begin{aligned}
b^{(\ell)}(n)= & \frac{1}{2} \sum_{j=0}^{2 M+1} E_{j}^{\ell}-\sum_{j=1}^{M-1} \mu_{j}(n)^{\ell} \\
& +\frac{\ell}{\pi} \int_{\sigma\left(H_{p}\right)} \lambda^{\ell-1} \arg \left(1+R_{ \pm}(\lambda) \frac{u_{ \pm}(\lambda, n)}{\overline{u_{ \pm}(\lambda, n)}}\right) d \lambda
\end{aligned}
$$

and in the special case $\ell=1$

$$
\begin{aligned}
b(n)= & \frac{1}{2} \sum_{j=0}^{2 M+1} E_{j}-\sum_{j=1}^{M-1} \mu_{j}(n) \\
& +\frac{1}{\pi} \int_{\sigma\left(H_{p}\right)} \arg \left(1+R_{ \pm}(\lambda) \frac{u_{ \pm}(\lambda, n)}{u_{ \pm}(\lambda, n)}\right) d \lambda .
\end{aligned}
$$


The analog of (7.24) in the case of Schrödinger operators with constant background and no eigenvalues was first derived in [11. The general case for Schrödinger operators can be found in 21. For further trace formulas in the constant background case, in particular in connection with the Toda lattice, we refer the reader to $[8]$, [16].

Remark 7.1. If $R_{ \pm}(\lambda)=0$ then $H$ can be obtained from $H_{p}$ by inserting the corresponding number of eigenvalues using the double commutation method provided in [20] since this transformation is easily seen to preserve the reflectionless property.

\section{ACKNOWLEDGMENTS}

I thank the referee for making several valuable suggestions.

\section{Appendix A. HeRglotz funCtions}

The results stated in this section can be found in [4 (see also [3]).

We set $\mathbb{C}_{ \pm}=\{z \in \mathbb{C} \mid \pm \operatorname{Im}(z)>0\}$. A function $F: \mathbb{C}_{+} \rightarrow \mathbb{C}_{+}$is called a Herglotz function (sometimes also Pick or Nevanlinna-Pick function), if $F$ is analytic in $\mathbb{C}_{+}$. For convenience one usually defines $F$ on $\mathbb{C}_{-}$by $F(\bar{z})=\overline{F(z)}$.

Herglotz functions can be characterized by

Theorem A.1. $F$ is a Herglotz function if and only if

$$
F(z)=a+b z+\int_{\mathbb{R}}\left(\frac{1}{\lambda-z}-\frac{\lambda}{1+\lambda^{2}}\right) d \rho(\lambda), \quad z \in \mathbb{C}_{+},
$$

where $a=\operatorname{Re}(F(\mathrm{i})) \in \mathbb{R}, b \geq 0$, and $\rho$ is a measure on $\mathbb{R}$ which satisfies $\int_{\mathbb{R}}(1+$ $\left.\lambda^{2}\right)^{-1} d \rho(\lambda)<\infty$.

Let $\ln (z)$ be defined such that $\ln (z)=\ln |z|+\mathrm{i} \arg (z),-\pi<\arg (z) \leq \pi$. Then $\ln (z)$ is holomorphic and $\operatorname{Im}(\ln (z))>0$ for $z \in \mathbb{C}_{+}$. Hence $\ln (z)$ is a Herglotz function.

The sum of two Herglotz functions is again a Herglotz function, similarly the composition of two Herglotz functions is Herglotz. In particular, if $F(z)$ is a Herglotz function, the same holds for $\ln (F(z))$ and $-\frac{1}{F(z)}$. Thus, using the representation A.1) for $\ln (F(z))$, we get another representation for $F(z)$.

Theorem A.2. (i). $F$ is a Herglotz function if and only if it has the representation

$$
F(z)=\exp \left\{c+\int_{\mathbb{R}}\left(\frac{1}{\lambda-z}-\frac{\lambda}{1+\lambda^{2}}\right) \xi(\lambda) d \lambda\right\}, \quad z \in \mathbb{C}_{+},
$$

where $c=\ln |F(\mathrm{i})| \in \mathbb{R}, \xi \in L^{1}\left(\mathbb{R},\left(1+\lambda^{2}\right)^{-1} d \lambda\right)$ real-valued and $\xi$ is not identically zero. Moreover,

$$
\xi(\lambda)=\frac{1}{\pi} \lim _{\varepsilon \downarrow 0} \operatorname{Im}(\ln (F(\lambda+\mathrm{i} \varepsilon)))=\frac{1}{\pi} \lim _{\varepsilon \downarrow 0} \arg (F(\lambda+\mathrm{i} \varepsilon))
$$

for a.e. $\lambda \in \mathbb{R}$, and $0 \leq \xi(\lambda) \leq 1$ for a.e. $\lambda \in \mathbb{R}$. Here $-\pi<\arg (F(\lambda+\mathrm{i} \varepsilon)) \leq \pi$ according to the definition of $\ln (z)$.

(ii). Fix $n \in \mathbb{N}$ and set $\xi_{+}(\lambda)=\xi(\lambda), \xi_{-}(\lambda)=1-\xi(\lambda)$. Then

$$
\int_{\mathbb{R}}|\lambda|^{n} \xi_{ \pm}(\lambda) d \lambda<\infty
$$


if and only if

$$
\int_{\mathbb{R}}|\lambda|^{n} d \rho(\lambda)<\infty \quad \text { and } \quad \lim _{z \rightarrow \mathrm{i} \infty} \pm F(z)= \pm a \mp \int_{\mathbb{R}} \frac{\lambda d \rho(\lambda)}{1+\lambda^{2}}>0 .
$$

(iii). We have

$$
F(z)= \pm 1+\int_{\mathbb{R}} \frac{d \rho(\lambda)}{\lambda-z} \text { with } \int_{\mathbb{R}} d \rho(\lambda)<\infty
$$

if and only if

$$
F(z)= \pm \exp \left( \pm \int_{\mathbb{R}} \xi_{ \pm}(\lambda) \frac{d \lambda}{\lambda-z}\right) \quad \text { with } \quad \xi_{ \pm} \in L^{1}(\mathbb{R})
$$

$\left(\xi_{ \pm}\right.$from above). In this case

$$
\begin{gathered}
\int_{\mathbb{R}} d \rho(\lambda)=\int_{\mathbb{R}} \xi_{ \pm}(\lambda) d \lambda . \\
\text { REFERENCES }
\end{gathered}
$$

[1] N. Akhiezer, The Classical Moment Problem, Oliver and Boyd, London, 1965.

[2] A. J. Antony and M. Krishna, Inverse spectral theory for Jacobi matrices and their almost periodicity, Proc. Indian Acad. Sci. (Math. Sci.) 104:4, 777-818 (1994).

[3] N. Aronszajn, On a problem of Weyl in the theory of singular Sturm-Liouville equations, Am. J. of Math. 79, 597-610 (1957).

[4] N. Aronszajn and W. Donoghue, On the exponential representation of analytic functions in the upper half-plane with positive imaginary part J. Analyse Mathematique 5, 321-388 (1956-57).

[5] F. Atkinson, Discrete and Continuous Boundary Problems, Academic Press, New York, 1964.

[6] J. Berezanskii, Expansions in Eigenfunctions of Self-adjoint Operators, Transl. Math. Monographs, vol. 17, Amer. Math. Soc., Providence, R. I., 1968.

[7] W. Bulla, F. Gesztesy, H. Holden, and G. Teschl Algebro-Geometric Quasi-Periodic Finite-Gap Solutions of the Toda and Kac-van Moerbeke Hierarchies, Memoirs of the Amer. Math. Soc. (to appear).

[8] K. M. Case, Orthogonal polynomials II, J. Math. Phys. 16, 1435-1440 (1975).

[9] K. M. Case and M. Kac, A discrete version of the inverse scattering problem, J. Math. Phys. 14, 594-603 (1973).

[10] E. Date and S. Tanaka, Analogue of inverse scattering theory for discrete Hill"s equations and exact solutions for the periodic Toda lattice, Prog. Th. Phys. 59, 457-465 (1976).

[11] P. Deift and E. Trubowitz, Inverse scattering on the line, Comm. Pure Appl. Math. 32, 121-251 (1979).

[12] H. Flaschka, Discrete and periodic illustrations of some aspects of the inverse method, in Dynamical Systems: Theory and Applications (ed. J. Moser), 441-466, Lecture Notes in Physics 38, Springer, Berlin, 1975.

[13] L. Fu and H. Hochstadt, Inverse theorems for Jacobi matrices, J. Math. Anal. Appl. 47, 162-168 (1974).

[14] I. M. Gel'fand and L. A. Dikii, Asymptotic behavior of the resolvent of Sturm-Liouville equations and the algebra of the Korteweg-de Vries equations, Russian Math. Surv. 30:5, 77-113 (1975).

[15] J. S. Geronimo and W. Van Assche, Orthogonal polynomials with asymptotically periodic recurrence coefficients, J. App. Th., 46, 251-283 (1986).

[16] F. Gesztesy and H. Holden, Trace formulas and conservation laws for nonlinear evolution equations, Rev. Math. Phys. 6, 51-95 (1994).

[17] F. Gesztesy and B. Simon, The xi-function, Acta Math. 176, 49-71 (1996).

[18] F. Gesztesy and B. Simon, Uniqueness theorems in inverse spectral theory for onedimensional Schrödinger operators, Trans. Amer. Math. Soc. 348, 349-373 (1996).

[19] F. Gesztesy and B. Simon, Rank one perturbations at infinite coupling, J. Funct. Anal. 128, 245-252 (1995). 
[20] F. Gesztesy and G. Teschl, Commutation methods for Jacobi operators, J. Diff. Eqs. 128, 252-299 (1996).

[21] F. Gesztesy, H. Holden, and B. Simon, Absolute summability of the trace relation for certain Schrödinger operators, Com. Math. Phys. 168, 137-161 (1995).

[22] F. Gesztesy, M. Krishna, and G. Teschl, On isospectral sets of Jacobi operators, Com. Math. Phys. 181, 631-645 (1996).

[23] F. Gesztesy ,R. Nowell, and W. Pötz, One-dimensional scattering for quantum systems with nontrivial spatial asymptotics, Diff. Integral Eqs. 10, 521-546 (1997).

[24] F. Gesztesy, R. Ratnaseelan, and G. Teschl, The KdV hierarchy and associated trace formulas, in Proceedings of the International Conference on Applications of Operator Theory, (eds. I. Gohberg, P. Lancaster, and P. N. Shivakumar), 125-163, Oper. Theory Adv. Appl., 87, Birkhäuser, Basel, 1996.

[25] F. Gesztesy, H. Holden, B. Simon, and Z. Zhao, Higher order trace relations for Schrödinger operators, Rev. Math. Phys. 7, 893-922 (1995).

[26] F. Gesztesy, H. Holden, B. Simon, and Z. Zhao, A trace formula for multidimensional Schrödinger operators, J. Funct. Anal. 141, 449-465 (1996).

[27] G. S. Guseinov, The inverse problem of scattering theory for a second-order difference equation on the whole axis, Soviet Math. Dokl., 17, 1684-1688 (1976).

[28] M. Klaus, On bound states of the infinite harmonic crystal, Hel. Phys. Acta 51, 793-803 (1978).

[29] M. G. Krein, Perturbation determinants and a formula for the traces of unitary and self-adjoint operators, Soviet Math. Dokl. 3, 707-710 (1962).

[30] I. M. Krichever, Algebro-geometric spectral theory of the Schrödinger difference operator and the Peierls model, Soviet Math. Dokl. 26, 194-198 (1982).

[31] B. M. Levitan, Inverse Sturm-Liouville Problems, VNU Science Press, Utrecht, 1987.

[32] P. van Moerbeke, The spectrum of Jacobi Matrices, Inv. Math. 37, 45-81 (1976).

[33] F. W. J. Olver, Asymptotics and Special Functions, Academic Press, New York, (1974).

[34] B. Simon, Spectral Analysis of Rank One Perturbations and Applications, proceedings, "Mathematical Quantum Theory II: Schrödinger Operators", (eds. J. Feldman, R. Froese, and L. M. Rosen), CRM Proc. Lecture Notes 8, 109-149 (1995).

[35] M.L. Sodin and P.M. Yuditskiŭ, Infinite-zone Jacobi matrices with pseudo-extendible Weyl functions and homogeneous spectrum, Russian Acad. Sci. Dokl. Math. 49, 364368 (1994).

[36] M. Sodin and P. Yuditskii, Almost periodic Jacobi matrices with homogeneous spectrum, infinite dimensional Jacobi inversion, and Hardy spaces of character-automorphic functions, preprint.

[37] G. Teschl, Oscillation theory and renormalized oscillation theory for Jacobi operators, J. Diff. Eqs. 129, 532-558 (1996).

[38] G. Teschl, Jacobi Operators and Completely Integrable Lattices, in preparation, available from http://www.mat.univie.ac.at/ gerald/ftp/book-jac/.

[39] M. Toda, Theory of Nonlinear Lattices, $2^{\text {nd }}$ enl. edition, Springer, Berlin, 1989.

Institut für Reine und Angewandte Mathematik, RWTH Aachen, 52056 Aachen, GerMANY

Current address: Institut für Mathematik, Strudlhofgasse 4, 1090 Wien, Austria

E-mail address: Gerald.Teschl@univie.ac.at

$U R L:$ http://www.mat.univie.ac.at/〜 gerald/ 